\title{
Stable Model Predictive Control for Constrained Max-Plus-Linear Systems
}

\author{
Ion Necoara - Bart De Schutter • \\ Ton J. J. van den Boom • Hans Hellendoorn
}

Received: 20 April 2006 / Accepted: 18 June 2007/

Published online: 21 July 2007

(C) Springer Science + Business Media, LLC 2007

\begin{abstract}
Discrete-event systems with synchronization but no concurrency can be described by models that are "linear" in the max-plus algebra, and they are called max-plus-linear (MPL) systems. Examples of MPL systems often arise in the context of manufacturing systems, telecommunication networks, railway networks, parallel computing, etc. In this paper we provide a solution to a finite-horizon model predictive control (MPC) problem for MPL systems where it is required that the closedloop input and state sequence satisfy a given set of linear inequality constraints. Although the controlled system is nonlinear, by employing results from max-plus theory, we give sufficient conditions such that the optimization problem that is performed at each step is a linear program and such that the MPC controller guarantees a priori stability and satisfaction of the constraints. We also show how one can use the results in this paper to compute a time-optimal controller for linearly constrained MPL systems.
\end{abstract}

Keywords Discrete-event systems • Max-plus-linear systems • Input-state constraints $\cdot$ Model predictive control $\cdot$ Stability $\cdot$ Positively invariant sets

This paper was not presented at any IFAC meeting.

I. Necoara $(\varangle)$ · B. De Schutter · T. J. J. van den Boom · H. Hellendoorn Delft Center for Systems and Control, Delft University of Technology, Mekelweg 2, 2628 CD Delft, Netherlands e-mail: i.necoara@yahoo.com

Present Address:

I. Necoara

Department of Electrical Engineering (ESAT),

Katholieke Universiteit Leuven, Kasteelpark Arenberg 10, bus 2446,

B-3001 Leuven-Heverlee, Belgium

e-mail: ion.necoara@esat.kuleuven.be 


\section{Introduction}

Discrete-event systems (DES) are event-driven dynamical systems (i.e. the state transitions are initiated by events, rather than a clock) and they often arise in the context of manufacturing systems, telecommunication networks, railway networks, parallel computing, etc. In Baccelli et al. (1992) it has been shown that a DES with synchronization but no concurrency can be modeled by a max-plus-linear (MPL) system. Although several authors have already developed methods to compute optimal controllers for MPL systems (Baccelli et al. 1992; Cofer and Garg 1996; De Schutter and van den Boom 2001; Kumar and Garg 1994; Libeaut and Loiseau 1995; Menguy et al. 1997, 2000; Necoara 2006), the literature on stabilizing controllers for this class of systems subject to input and state constraints is relatively sparse. Some of the contributions that partially address this problem include model predictive control (MPC; De Schutter and van den Boom 2001, 2006) and optimal control based on residuation theory (Cottenceau et al. 2001; Maia et al. 2003; Menguy et al. 1997, 2000). In Maia et al. (2003) an optimal controller is derived based on residuation theory that guarantees also stability. However, the residuation-based approach does not cope with input and state constraints. Moreover, the methods presented in Menguy et al. (1997) and Cottenceau et al. (2001) cannot solve tracking problems corresponding to the case when the actual outputs do not necessarily have to occur before the due dates although these situations are often met in many practical applications. Some of these drawbacks are removed in Maia et al. (2003), Menguy et al. (2000), and Necoara (2006) by using respectively projection, an adaptive approach, or MPC. The main difference between our approach and the papers mentioned previously is that in those papers the optimal controller does not satisfy both requirements, i.e. a priori stability of the closed-loop system and that the closed-loop input and state sequence should satisfy a given set of linear inequality constraints.

MPC (Maciejowski 2002; Mayne et al. 2000) is one of the most applied advanced control technique in the process industry. MPC provides many attractive features: it is an easy-to-tune method, it is applicable to multi-variable systems, it can handle constraints, and it is capable of tracking pre-scheduled reference signals. The essence of MPC is to determine a control profile that optimizes a cost criterion over a prediction window and then to apply this control profile until new process measurements become available when the whole procedure is repeated. Feedback is incorporated by using those measurements to update the optimization problem for the next step.

This paper considers the problem of designing a stabilizing MPC scheme for the class of MPL systems where the input and state sequence must satisfy a given set of linear inequality constraints. We follow here a similar finite-horizon MPC approach as the one developed in Maciejowski (2002) and Mayne et al. (2000) for conventional, time-driven systems and that uses a terminal set and a terminal cost as basic ingredients. However, the extension from classical time-driven systems to discrete-event MPL systems is not trivial since many concepts from system theory have to be adapted adequately. One of the key results of the paper is to provide sufficient conditions based on a terminal set and a terminal cost approach such that one can compute an MPC controller that guarantees a priori stability and constraint satisfaction for the closed-loop MPL system. 
The paper is organized as follows. In Section 2 we introduce some notation, and we give a short introduction to MPL systems. We also formulate the control problem that we are going to solve in this paper. We also introduce the notion of (Lyapunov) stability for MPL systems. Moreover, we will see that under some additional assumptions Lyapunov stability of the closed-loop MPC also implies stability in terms of boundedness of the buffer levels as defined in Baccelli et al. (1992) and Passino and Burgess (1998). In Section 3 we define the concept of positively invariant set for MPL systems and we derive the main properties of such a set. We show that under mild assumptions the maximal positively invariant set is a polyhedron. In Section 4 we propose an MPC scheme based on a terminal setterminal cost approach that guarantees a priori stability of the closed-loop system and also that the input-state constraints are not violated. We show that for certain nonnegative piecewise affine stage costs the optimization problem that is solved at each step can be recast as a linear program. In Section 5 we formulate the timeoptimal control problem for constrained MPL systems in a slightly different fashion from the classical one and we provide a solution based on linear programming. Next, in Section 6 we illustrate the method proposed in this paper with an example. Section 7 concludes the paper.

\section{Preliminaries}

\subsection{Notation}

We define $\varepsilon:=-\infty, \mathbb{R}_{\varepsilon}:=\mathbb{R} \cup\{\varepsilon\}$, and $\mathbb{R}_{+}=\{x \in \mathbb{R}: x \geq 0\}$. The max-plusalgebraic (MPA) addition $(\oplus)$ and multiplication $(\otimes)$ are defined as (Baccelli et al. 1992; Heidergott et al. 2005)

$$
x \oplus y:=\max \{x, y\}, \quad x \otimes y:=x+y \quad \text { for } \quad x, y \in \mathbb{R}_{\varepsilon} .
$$

For matrices $A, B \in \mathbb{R}_{\varepsilon}^{m \times n}$ and $C \in \mathbb{R}_{\varepsilon}^{n \times p}$ one can extend the definition as follows:

$$
(A \oplus B)_{i j}:=A_{i j} \oplus B_{i j}, \quad(A \otimes C)_{i j}:=\bigoplus_{k=1}^{n} A_{i k} \otimes C_{k j} \quad \text { for all } i, j .
$$

Define the matrix $\varepsilon$ as the MPA zero matrix of appropriate dimension: $\varepsilon_{i j}:=\varepsilon$ for all $i, j$. The matrix $E$ is the MPA identity matrix: $E_{i i}:=0$ for all $i$ and $E_{i j}:=\varepsilon$ for all $i, j$ with $i \neq j$. Let $k$ be a nonnegative integer. Then for any square matrix $A$ the $k$ th MPA power of $A$ is defined by $A^{{ }^{k}}:=A \otimes A \otimes \cdots \otimes A$ ( $k$ times) if $k>0$, and $A^{\otimes^{0}}=E$. We define $A^{*}$, whenever it exists, by $A^{*}:=\lim _{k \rightarrow \infty} E \oplus A \oplus \cdots \oplus A^{\otimes^{k}}$. For a given matrix $H$, by $H \geq 0$ we mean that $H$ is nonnegative, i.e. $H_{i j} \geq 0$ for all $i, j$. We use $\mathbb{N}$ to denote the set of nonnegative integers. For $k, l \in \mathbb{N}$ with $k \leq l$, $\mathbb{N}_{[k, l]}$ represents the set $\{k, k+1, \cdots, l\}$. A matrix $\Gamma \in \mathbb{R}_{\varepsilon}^{n \times m}$ is row-finite if for any row $i \in \mathbb{N}_{[1, n]}$, we have $\max _{j \in \mathbb{N}_{[1, m]}} \Gamma_{i j} \neq \varepsilon$; a column-finite matrix is defined similarly. Throughout the paper $\|\cdot\|_{\infty}$ represents the $\infty$-norm $\left(\|x\|_{\infty}:=\max _{i \in \mathbb{N}_{[1, n]}}\left|x_{i}\right|\right.$ for $\left.x \in \mathbb{R}^{n}\right)$. Let $d_{\infty}$ denote the metric on $\mathbb{R}^{n}$ induced by the $\infty$-norm. Given a closed set $\mathcal{X} \subseteq \mathbb{R}^{n}$ and a point $x \in \mathbb{R}^{n}$ then $d_{\infty}(x, \mathcal{X}):=\min _{y \in \mathcal{X}}\|x-y\|_{\infty}$ denotes the distance 
from $x$ to $\mathcal{X}$. For $\in \mathbb{R}_{\varepsilon}^{n}$ we define $\|x\|_{\oplus}:=\max \left\{x_{1}, \ldots, x_{n}\right\}$. For a vector $x \in \mathbb{R}_{\varepsilon}^{n}$ and a scalar $\lambda \in \mathbb{R}_{\varepsilon}$, we define $\lambda \otimes x:=x+\lambda:=\left[x_{1}+\lambda \ldots x_{n}+\lambda\right]^{T}$ (for a matrix $A$, $\lambda \otimes A:=A+\lambda$ is defined similarly).

We denote with $x \oplus^{\prime} y:=\min \{x, y\}$ and $x \otimes^{\prime} y:=x+y$ (the operations $\otimes$ and $\otimes^{\prime}$ differ only in that $(-\infty) \otimes(+\infty):=-\infty$, while $\left.(-\infty) \otimes^{\prime}(+\infty):=+\infty\right)$. The matrix multiplication and addition for $\left(\oplus^{\prime}, \otimes^{\prime}\right)$ are defined similarly as for $(\oplus, \otimes)$. It is known (see e.g. Baccelli et al. 1992, Chapter 4) that the following inequalities hold for any matrix $A$ and vectors $x, y$ of appropriate dimensions over $\mathbb{R}_{\varepsilon}$ :

$$
x \leq y \Rightarrow A \otimes x \leq A \otimes y \text { and } A \otimes^{\prime} x \leq A \otimes^{\prime} y,
$$

where we consider the partial order defined by the positive orthant cone (i.e. $x \leq y$ if and only if (iff) $x_{i} \leq y_{i}$ for all $i$ ). The following results are well-known in max-plus algebra (Baccelli et al. 1992, Section 3.2.3):

\section{Result 2.1}

(i) The inequality $A \otimes x \leq b$ in max-algebra has the largest solution given by $x_{\mathrm{opt}}=$ $\left(-A^{T}\right) \otimes^{\prime} b$ (by the largest solution we mean that for all $x$ satisfying $A \otimes x \leq b$ we have $\left.x \leq x_{\mathrm{opt}}\right)$.

(ii) The equation $x=A \otimes x \oplus b$ has $x=A^{*} \otimes b$ as a solution. If $A_{i j}<0$ for all $i$, $j$, then this solution is unique.

In this paper we use both max-plus and conventional algebra. Therefore, we will always write the operators " $\oplus$ " and " $\otimes$ " explicitly. The operators "+" and "." denote the conventional summation and multiplication operators (the "." operator is usually omitted, except for mixed equations where we want to stress that a multiplication in conventional algebra is involved). We also use mixed properties like distributivity of + with respect to $\oplus$, i.e., $x+(y \oplus z)=(x+y) \oplus(x+z)$ for $x, y, z, \in \mathbb{R}_{\varepsilon}$, and mixed associativity, i.e., $x+(y \otimes z)=(x+y) \otimes z)$ for $x, y, z, \in \mathbb{R}_{\varepsilon}$, which imply that

$$
(A+\lambda) \otimes(x+\mu)=(A \otimes x)+(\lambda+\mu)
$$

for all scalars $\lambda, \mu$ and a vector $x$ and matrix $A$ of appropriate dimensions.

\subsection{Max-plus-linear systems}

An MPL system is defined as follows (Baccelli et al. 1992; Cuninghame-Green 1979; Heidergott et al. 2005):

$$
x_{\text {sys }}(k):=A_{\text {sys }} \otimes x_{\text {sys }}(k-1) \oplus B_{\text {sys }} \otimes u_{\text {sys }}(k), \quad y_{\text {sys }}(k):=C_{\text {sys }} \otimes x_{\text {sys }}(k),
$$

where $x_{\text {sys }}(k) \in \mathbb{R}_{\varepsilon}^{n}$ represents the state, $u_{\text {sys }}(k) \in \mathbb{R}_{\varepsilon}^{m}$ is the input, $y_{\text {sys }}(k) \in \mathbb{R}_{\varepsilon}^{p}$ is the output and where $A_{\text {sys }} \in \mathbb{R}_{\varepsilon}^{n \times n}, B_{\text {sys }} \in \mathbb{R}_{\varepsilon}^{n \times m}, C_{\text {sys }} \in \mathbb{R}_{\varepsilon}^{p \times n}$ are the system matrices. ${ }^{1}$ In the context of DES $k$ is an event counter while $u_{\text {sys }}, x_{\text {sys }}$ and $y_{\text {sys }}$ are dates (feeding times, processing times and finishing times, respectively). Note that for MPL systems at the $k$ th event the feeding time $u_{\text {sys }}(k)$ has direct influence on the processing time

\footnotetext{
${ }^{1}$ We may assume without loss of generality that $B_{\text {sys }}$ is column-finite and $C_{\text {sys }}$ is row-finite, since otherwise the corresponding inputs and outputs can be eliminated from the description model. 
$x_{\text {sys }}(k)$ (see also Section 6). The monotonicity property of the max operator Eq. 1 implies that the MPL systems are a particular class of monotone systems.

The scalar $\lambda \in \mathbb{R}_{\varepsilon}$ is an MPA eigenvalue of the matrix $A$ if there exists a vector $v \in \mathbb{R}_{\varepsilon}^{n}$ with at least one finite entry such that $A_{\text {sys }} \otimes v=\lambda \otimes v$ (Baccelli et al. 1992; Heidergott et al. 2005). In the sequel we use $\lambda_{\max }$ to denote the maximal MPA eigenvalue of $A_{\text {sys. }}$. In practice, the finite entries of the system matrix $A_{\text {sys }}$ will always be nonnegative as they correspond to processing and transportation times. This implies that in practice $\lambda_{\max } \geq 0$.

In this paper we consider a reference signal (i.e. a due date signal) that the output should track of the form:

$$
r_{\text {sys }}(k):=y_{\mathrm{t}}+k \rho,
$$

with $y_{\mathrm{t}} \in \mathbb{R}^{p}$. In practice, such a reference signal is often used as it corresponds to a regular and smooth due date signal with a constant output rate. In a manufacturing context, this would correspond to situation with a steady production rate where we have to produce a new product every $\rho$ time units. Note that we can also consider a more general signal $r_{\text {sys }}$ such that there exists a finite positive integer $K^{\mathrm{r}}$ for which $r_{\mathrm{sys}}(k)=y_{\mathrm{t}}+k \rho$ for all $k \geq K^{\mathrm{r}}$. The subsequent derivations will then remain the same.

Since time is not scalable, typical constraints for an MPL system (Eq. 3) are

$$
\begin{array}{ll}
y_{\text {sys }}(k) \leq r_{\text {sys }}(k)+h^{\mathrm{yu}}, & \left(u_{\text {sys }}\right)_{i}(k)-\left(u_{\text {sys }}\right)_{j}(k) \leq h_{i j}^{\mathrm{u}}, \\
\left(x_{\text {sys }}\right)_{i}(k)-\left(u_{\text {sys }}\right)_{j}(k) \leq h_{i j}^{\mathrm{xu}}, & u_{\text {sys }}(k+1)-u_{\text {sys }}(k) \geq 0 .
\end{array}
$$

The constraint $u_{\text {sys }}(k+1)-u_{\text {sys }}(k) \geq 0$ appears in the context of DES where the input represents times, so the input sequence should be nondecreasing. Moreover, the constraints $\left(u_{\text {sys }}\right)_{i}(k)-\left(x_{\text {sys }}\right)_{j}(k) \leq h_{i j}^{\text {ux }}$ are implicitly defined by the MPL system. Note that, in general, the constraint $\left(x_{\text {sys }}\right)_{i}(k)-\left(x_{\text {sys }}\right)_{j}(k) \leq h_{i j}^{\mathrm{x}}$ can be satisfied (with some conservativeness) if a constraint of the type $\left(x_{\text {sys }}\right)_{i}(k)-\left(u_{\text {sys }}\right)_{j}(k) \leq h_{i j}^{\mathrm{xu}}$ is fulfilled. The constraints 5 and 6 can be generalized as follows:

$$
\begin{aligned}
& H_{\text {sys }} x_{\text {sys }}(k)+G_{\text {sys }} u_{\text {sys }}(k) \leq h_{\text {sys }}(k) \\
& u_{\text {sys }}(k+1)-u_{\text {sys }}(k) \geq 0
\end{aligned}
$$

where $H_{\text {sys }} \geq 0$. Later on we will propose methods to compute input signals that satisfy these constraints. Note that the constraint 8 does not fit the form Eq. 7 . However, we can include Eq. 8 into Eq. 7 as follows: we introduce a new state vector $\bar{x}_{\text {sys }}(k)=\left[\begin{array}{lll}x_{\text {sys }}^{T}(k) & z^{T}(k)\end{array}\right]^{T}$ with the dynamics

$$
\begin{aligned}
& \bar{x}_{\text {sys }}(k)=\bar{A}_{\text {sys }} \otimes \bar{x}_{\text {sys }}(k-1) \oplus \bar{B}_{\text {sys }} \otimes u_{\text {sys }}(k) \\
& \bar{y}_{\text {sys }}(k)=\bar{C}_{\text {sys }} \otimes \bar{x}_{\text {sys }}(k)
\end{aligned}
$$

and the extra constraint:

$$
\left[\begin{array}{ll}
0 & I_{m}
\end{array}\right] \bar{x}_{\text {sys }}(k) \leq u_{\text {sys }}(k),
$$

with $\bar{A}_{\text {sys }}=\left[\begin{array}{cc}A_{\text {sys }} & B_{\text {sys }} \\ \varepsilon & E\end{array}\right], \bar{B}_{\text {sys }}=\left[\begin{array}{c}B_{\text {sys }} \\ E\end{array}\right]$ and $\bar{C}_{\text {sys }}=\left[\begin{array}{ll}C_{\text {sys }} & \varepsilon\end{array}\right]$, and where $I_{m}$ denotes the $m \times m$ identity matrix in conventional algebra. Given the initial conditions $x_{\text {sys }}(0)$ and $u_{\text {sys }}(0)$ for the system 3 with constraints 7 and 8 and the initial conditions 
$\bar{x}_{\text {sys }}(0)=\left[x_{\text {sys }}(0)^{T} u_{\text {sys }}(0)^{T}\right]^{T}$ and $u_{\text {sys }}(0)$ for the new system 9 and 10 with the extra constraint 11 then by applying the same input signal $u_{\text {sys }}$ (which should satisfy Eq. 11) to both systems we obtain that the first $n$ components of $\bar{x}_{\text {sys }}(k)$ coincide with $x_{\text {sys }}(k)$ and the last $m$ components of $\bar{x}_{\text {sys }}(k)$ coincide with $u_{\text {sys }}(k)$. Note that the constraints 7 and 8 corresponding to the MPL system 3 can be written for the new system (Eqs. 9 and 10$)$ as $\left[H_{\text {sys }} 0\right] \bar{x}_{\text {sys }}(k)+G_{\text {sys }} u_{\text {sys }}(k) \leq h_{\text {sys }}(k)$ and the extra constraint 11 as $\left[\begin{array}{ll}0 & I_{m}\end{array}\right] \bar{x}_{\text {sys }}(k)-I_{m} u_{\text {sys }}(k) \leq 0$, i.e.

$$
\bar{H}_{\text {sys }} \bar{x}_{\text {sys }}(k)+\bar{G}_{\text {sys }} u_{\text {sys }}(k) \leq \bar{h}_{\text {sys }}(k),
$$

where $\bar{H}_{\text {sys }}=\left[\begin{array}{cc}H_{\text {sys }} & 0 \\ 0 & I_{m}\end{array}\right], \bar{G}_{\text {sys }}=\left[\begin{array}{c}G_{\text {sys }} \\ -I_{m}\end{array}\right]$ and $\bar{h}_{\text {sys }}(k)=\left[\begin{array}{c}h_{\text {sys }}(k) \\ 0\end{array}\right]$. Note that the property $H_{\text {sys }} \geq 0$ is preserved under the previous transformation, i.e. $\bar{H}_{\text {sys }} \geq 0$.

Recall that the maximal MPA eigenvalue $\lambda_{\max }$ of $A_{\text {sys }}$ is in practice always nonnegative. Since $\bar{A}_{\text {sys }}$ has an upper diagonal block structure and since the MPA eigenvalue of $E$ is 0 , the maximal MPA eigenvalue of $\bar{A}_{\text {sys }}$ is given by $\max \left\{\lambda_{\max }, 0\right\}=$ $\lambda_{\max }$. Since the maximal MPA eigenvalue of the system matrix $\bar{A}_{\text {sys }}$ characterizes the maximal throughput of the system (Eqs. 9 and 10; see e.g. Baccelli et al. 1992, Section 3.7) and since through the term $\bar{B}_{\text {sys }} \otimes u_{\text {sys }}$ it is possible to create delays in the starting times of activities, we should choose a slope $\rho$ for the reference signal such that $\rho \geq \lambda_{\max }$. Since $\lambda_{\max }$ is finite in practice, there exists an MPA invertible matrix $P \in \mathbb{R}_{\varepsilon}^{n \times n}$ such that the matrix $\bar{A}=P^{\otimes^{-1}} \otimes \bar{A}_{\text {sys }} \otimes P$ satisfies $\bar{A}_{i j} \leq \lambda_{\text {max }}$ for all $i, j \in \mathbb{N}_{[1, n]}$ (see ${ }^{2}$ e.g. De Schutter 1996; Gaubert 1995; Mairesse 1996), where $P^{\otimes^{-1}}$ denotes the inverse of the matrix $P$ in the max-plus algebra, i.e. $P^{\otimes^{-1}} \otimes P=$ $P \otimes P^{\otimes^{-1}}=E$. We make the following change of coordinates: $\bar{x}(k)=P^{\otimes^{-1}} \otimes \bar{x}_{\text {sys }}(k)$. We denote with $\bar{B}=P^{\otimes^{-1}} \otimes \bar{B}_{\text {sys }}, \bar{C}=\bar{C}_{\text {sys }} \otimes P$ and $\bar{y}(k)=\bar{y}_{\text {sys }}(k), \bar{u}(k)=u_{\text {sys }}(k)$. In the new coordinates the system (Eqs. 9 and 10) becomes:

$$
\bar{x}(k)=\bar{A} \otimes \bar{x}(k-1) \oplus \bar{B} \otimes \bar{u}(k), \quad \bar{y}(k)=\bar{C} \otimes \bar{x}(k) .
$$

If we define $x(k)=\bar{x}(k)-\rho k, u(k)=\bar{u}(k)-\rho k, y(k)=\bar{y}(k)-\rho k, A=\bar{A}-\rho$ (i.e. we subtract in the conventional algebra from all entries of $\bar{x}, \bar{u}, \bar{y}$ and $\bar{A}$ the values $\rho k$ and $\rho$, respectively) and $B=\bar{B}, C=\bar{C}$, we obtain the normalized system corresponding to the original system (Eq. 3). Using Eq. 2 it follows that this normalized system can be written as

$$
\begin{aligned}
& x(k)=A \otimes x(k-1) \oplus B \otimes u(k) \\
& y(k)=C \otimes x(k) .
\end{aligned}
$$

Input and output signals determined for this normalized system can be transformed into signals for the original system by adding the signal $\rho k$ (i.e., by applying the

\footnotetext{
${ }^{2}$ In (Mairesse 1995, Lemma 3) and (Gaubert 1996, Lemma 4.8) the matrix $P$ is constructed as follows for an irreducible matrix $A_{\text {sys }}: P_{i i}=v_{i}$ for all $i$ and $P_{i j}=\varepsilon$ for all $i$, with $i \neq j$, where $v$ is an MPA

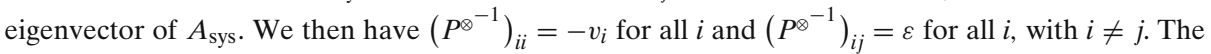
extension to a reducible matrix $A_{\text {sys }}$ can be done in a similar fashion (see e.g. De Schutter 1996, Section C.2). Note that in fact these results are related to similar results in the theory of Hadamard products of nonnegative matrices in conventional algebra (see Elsner et al. 1988, Theorem 7).
}

空 Springer 
inverse transformation). Note that $A<0$ if $\rho>\lambda_{\max }$, and that the maximal MPA eigenvalue of $A$ is $\lambda_{\max }-\rho<0$. In the sequel we will consider only MPL systems in the form of Eqs. 13 and 14, with $A \in \mathbb{R}_{\varepsilon}^{n \times n}, B \in \mathbb{R}_{\varepsilon}^{n \times m}, C \in \mathbb{R}_{\varepsilon}^{p \times n}$ and where the matrix $A$ satisfies $A<0$ if $\rho>\lambda_{\max }$. We frequently use the short-hand notation

$$
f(x, u):=A \otimes x \oplus B \otimes u .
$$

The MPL system (Eqs. 13 and 14) is controllable iff each component of the state can be made arbitrarily large by applying an appropriate controller to the system initially at rest. It follows [see Theorem 3.2 in Gazarik and Kamen (1999)] that the system is controllable iff the matrix $\Gamma:=\left[B A \otimes B \cdots A^{\otimes^{n-1}} \otimes B\right]$ is row-finite [note that this definition is equivalent to the one given in Baccelli et al. (1992) and Gazarik and Kamen (1999) where the system is controllable if all states are connected to some input). Similarly, the system (Eqs. 13 and 14) is observable iff each state is connected to some output, i.e. the matrix $\Omega:=\left[C^{T}(C \otimes A)^{T} \cdots\left(C \otimes A^{\otimes^{n-1}}\right)^{T}\right]^{T}$ is columnfinite [see Theorem 3.9 in Gazarik and Kamen (1999)] .

For the MPL system (Eqs. 13 and 14) the following key assumptions will be used throughout the paper:

A1: We assume that $\rho>\lambda_{\max } \geq 0$ (and thus $A<0$ ), and that the system is controllable and observable.

A2: There exist matrices $H \geq 0, G$ and a vector $h$ of appropriate dimensions such that the constraints (Eq. 12) can be written for the normalized system (Eqs. 13 and 14) as

$$
H x(k)+G u(k) \leq h .
$$

The conditions from Assumptions A1-A2 are quite weak and are usually met in applications. Note that $\rho$ can be chosen arbitrarily close to $\lambda_{\max }$ (see also the previous discussion). Moreover, since we consider constraints of the form of Eqs. 5 and 6, it follows that $h$ does not depend on $k$.

In the new coordinates the output should be regulated to the desired target $y_{\mathrm{t}}$. From Assumption A1 it follows that $A_{i j}<0$ for all $i, j \in \mathbb{N}_{[1, n]}$ and so [see Baccelli et al. (1992, Theorem 3.20) or Heidergott et al. (2005, Section 2.3)]

$$
A^{*}=E \oplus A \oplus \cdots \oplus A^{\otimes^{n-1}} .
$$

For any finite vector $u$ there exists a state equilibrium $x$, i.e. $x=A \otimes x \oplus B \otimes u$, given by $x=A^{*} \otimes B \otimes u$. Note that $x$ is unique [according to Result 2.1 (ii)] and finite (since $\Gamma$ is row-finite). We associate to $y_{\mathrm{t}}$ the largest equilibrium pair $\left(x_{\mathrm{e}}, u_{\mathrm{e}}\right)$ satisfying $^{3} C \otimes x_{\mathrm{e}} \leq y_{\mathrm{t}}$. From the previous discussion it follows that $\left(x_{\mathrm{e}}, u_{\mathrm{e}}\right)$ is given by

$$
u_{\mathrm{e}}:=\left(-\left(C \otimes A^{*} \otimes B\right)\right)^{T} \otimes^{\prime} y_{\mathrm{t}}, \quad x_{\mathrm{e}}:=A^{*} \otimes B \otimes u_{\mathrm{e}} .
$$

Since we may assume that $B_{\text {sys }}$ is column-finite and $C_{\text {sys }}$ is row-finite (see Footnote 1 ) and since the system is controllable and observable by Assumption A1, every input

\footnotetext{
${ }^{3}$ By the largest pair we mean that any other feasible equilibrium pair $(x, u)$ satisfies $x \leq x_{\mathrm{e}}, u \leq u_{\mathrm{e}}$. Moreover, we impose $C \otimes x_{\mathrm{e}} \leq y_{\mathrm{t}}$ since in applications it is preferable that the products be delivered in time once the steady (periodic) behavior is reached.
} 
of the system will influence some output, which implies that $C \otimes A^{*} \otimes B$ is columnfinite. As a consequence, $u_{\mathrm{e}}$ is finite. Hence, $x_{\mathrm{e}}$ is also finite. Note that in fact $\left(x_{\mathrm{e}}, u_{\mathrm{e}}\right)$ depends on the reference signal, but for the sake of simplicity of notation we drop this dependence.

\subsection{Stability for MPL systems}

In this section we adopt the formulation developed in (Necoara 2006; Passino and Burgess 1998; La Salle 1976) to the study of stability of MPL systems. We use the symbol $\mathbf{u}$ to denote a control sequence ${ }^{4}$ and $\phi(k ; x, \mathbf{u})$ to denote the state solution of Eq. 13 at event step $k$ when the initial state is $x$ at event step 0 and the control sequence $\mathbf{u}$ is applied. By definition $\phi(0 ; x, \mathbf{u}):=x$. For a state feedback law $\kappa: \mathbb{R}^{n} \rightarrow \mathbb{R}^{m}$ applied to Eqs. 13 and 14 we study the stability properties of the closed-loop system:

$$
x(k)=A \otimes x(k-1) \oplus B \otimes \kappa(x(k-1)), \quad y(k)=C \otimes x(k) .
$$

Similarly to the notation $\phi(k ; x, \mathbf{u})$, we denote by $\phi(k ; x, \kappa)$ the state solution of Eq. 18 at step $k$ when the initial state is $x$ at event step 0 and the feedback law $\kappa$ is applied.

Definition 2.2 The set $X_{\mathrm{e}} \subseteq \mathbb{R}^{n}$ is called positively invariant for Eq. 18 if for all $x \in X_{\mathrm{e}}$ it follows that $\phi(k ; x, \kappa) \in X_{\mathrm{e}}$ for all $k \geq 0$.

Definition 2.3 A closed positively invariant set $X_{\mathrm{e}}$ is called stable (Lyapunov stable as it is sometimes called) for the system 18 if for any $\theta>0$ there exists a $\delta>0$ such that for all $x$ satisfying $d_{\infty}\left(x, X_{\mathrm{e}}\right)<\delta$ we have $d_{\infty}\left(\phi(k ; x, \kappa), X_{\mathrm{e}}\right)<\theta$ for all $k \geq 0$.

If in addition to being stable, we have $d_{\infty}\left(\phi(k ; x, \kappa), X_{\mathrm{e}}\right) \rightarrow 0$ as $k \rightarrow \infty$ for all $x \in X$, then $X_{\mathrm{e}}$ is asymptotically stable for Eq. 18. In this case $X$ is called a region of attraction.

Remark 2.4 In Baccelli et al. (1992), Passino and Burgess (1998), and Necoara (2006) stability for DES is defined in terms of boundedness of the buffer levels (i.e. there exists a finite $M>0$ such that at any time the number of parts in any buffer is less than $M)$. Let us note that our definition of stability implies in particular that for any $x \in \mathbb{R}^{n},\left\|\phi(k ; x, \kappa)-x_{\mathrm{e}}\right\|_{\infty}$ is bounded for all $k \geq 0$, whenever the set $X_{\mathrm{e}}$ is bounded. For a controllable and observable system the boundedness of the state trajectory implies also boundedness of the output and of the input, i.e. $\left\|y(k)-y_{\mathrm{t}}\right\|_{\infty}$ and $\left\|u(k)-u_{\mathrm{e}}\right\|_{\infty}$ are bounded as well for all $k \geq 0$. For the original system boundedness of the state trajectory implies $\left\|x_{\text {sys }}(k)-\rho k\right\|_{\infty},\left\|y_{\text {sys }}(k)-\rho k\right\|_{\infty}$ and $\left\|u_{\text {sys }}(k)-\rho k\right\|_{\infty}$ are bounded for all $k \geq 0$ which leads to boundedness of the buffer levels ${ }^{5}$ [see also Passino and Burgess 1998, Definition 3.5].

We now introduce the so-called $\mathcal{K}$-functions: a function $\alpha: \mathbb{R}_{+} \rightarrow \mathbb{R}_{+}$is said to be a $\mathcal{K}$-function if (i) $\alpha(0)=0$, (ii) $\alpha(z)>0$ for all $z>0$, and (iii) $\alpha$ is strictly increasing.

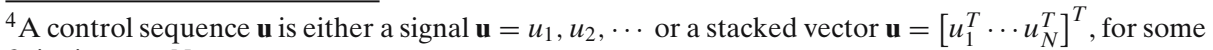
finite integer $N$.

${ }^{5}$ See Necoara et al. (2006) for a formal proof.

Springer
} 
The following theorem gives sufficient conditions for asymptotic stability of the system 18 .

Theorem 2.5 Let $X$ be a positively invariant set for the system 18 . Let $V: X \rightarrow \mathbb{R}$ be a function and let $X_{\mathrm{e}}$ be a closed subset of the interior of $X$ such that

(i) $V(x)=0$ for all $x \in X_{\mathrm{e}}$, and $V$ is continuous on a neighborhood of $X_{\mathrm{e}}$, and

(ii) $V(x) \geq \alpha\left(d_{\infty}\left(x, X_{\mathrm{e}}\right)\right)$ for all $x \in X$, where $\alpha$ is a $\mathcal{K}$-function, and

(iii) $V(f(x, \kappa(x)))-V(x) \leq-\beta\left(d_{\infty}\left(x, X_{\mathrm{e}}\right)\right)$ for all $x \in X$, where $\beta$ is a $\mathcal{K}$-function.

Then, $X_{\mathrm{e}}$ is asymptotically stable for Eq. 18 with a region of attraction $X$.

Proof In Necoara (2006, Corollary C.1.4) a proof is given for the case $X_{\mathrm{e}}=\left\{x_{\mathrm{e}}\right\}$, i.e. the equilibrium point. However, following exactly the same steps, this proof can be extended to the case of a general set $X_{\mathrm{e}}$ [see e.g. Passino and Burgess (1998, Theorem 3.2) or La Salle (1976, Theorem 7.9)].

We formulate now the control problem that we solve in the sequel:

Problem definition: Given the MPL system (Eqs. 13 and 14), a reference signal of the form of Eq. 4, and constraints of the form of Eq. 15, design a state feedback law $\kappa(x)$ such that the closed-loop system is asymptotically stable with respect to some closed positively invariant set $X_{\mathrm{e}}$ and such that the constraints 15 are satisfied.

\section{Positively invariant sets for MPL systems}

\subsection{Properties of the equilibrium pair $\left(x_{\mathrm{e}}, u_{\mathrm{e}}\right)$}

Recall that the equilibrium pair $\left(x_{\mathrm{e}}, u_{\mathrm{e}}\right)$ defined in Eq. 17 is finite. Furthermore, we assume that $\left(x_{\mathrm{e}}, u_{\mathrm{e}}\right)$ belongs to the set described by the constraints 15 , i.e. $\{(x, u)$ : $H x+G u \leq h\}$ (if this is not the case we determine $\left(x_{\mathrm{e}}, u_{\mathrm{e}}\right)$ as the optimal solution of the following linear programming problem: $\max _{u}\left\{\sum_{i=1}^{m} u_{i}: x=A^{*} \otimes B \otimes u\right.$, $\left.\left.C \otimes x \leq y_{\mathrm{t}}, H x+G u \leq h\right\}\right)$. We now consider the following MPL system:

$$
x(k)=A \otimes x(k-1) \oplus B \otimes u_{\mathrm{e}}, \quad y(k)=C \otimes x(k) .
$$

First let us show that $X_{\mathrm{e}}=\left\{x_{\mathrm{e}}\right\}$ is asymptotically stable for Eq. 19 with a region of attraction $\mathbb{R}_{\varepsilon}^{n}$. Before proving this statement let us note that from the property of non-expansiveness (see e.g. Heidergott et al. 2005, Lemma 3.10) it follows that

$$
\|(A \otimes x \oplus B \otimes u)-(A \otimes y \oplus B \otimes v)\|_{\infty} \leq\|x-y\|_{\infty} \oplus\|u-v\|_{\infty}
$$

for any matrices $A \in \mathbb{R}_{\varepsilon}^{n \times n}$ and $B \in \mathbb{R}_{\varepsilon}^{n \times m}$ such that $\left[\begin{array}{ll}A & B\end{array}\right]$ is row-finite and for any $x, y \in \mathbb{R}^{n}$ and $u, v \in \mathbb{R}^{m}$.

Theorem 3.1 Suppose that Assumption A1 holds and the equilibrium pair $\left(x_{\mathrm{e}}, u_{\mathrm{e}}\right)$ is finite. Then, the set $X_{\mathrm{e}}=\left\{x_{\mathrm{e}}\right\}$ is asymptotically stable with respect to the closed-loop system 19 with $\mathbb{R}^{n}$ as region of attraction. Moreover, the convergence towards $\left\{x_{\mathrm{e}}\right\}$ is achieved in a finite number of steps. 
Proof Note that $\phi\left(k ; x, u_{\mathrm{e}}\right)=A^{\otimes^{k}} \otimes x \oplus\left(\bigoplus_{t=1}^{k} A^{\otimes^{k-t}} \otimes B \otimes u_{\mathrm{e}}\right)$. Recall that $A_{i j}<0$ for all $i, j \in \mathbb{N}_{[1, n]}$ (according to Assumption A1). Then it is well-known (see e.g. Heidergott et al. 2005, Section 2.3) that for all $x \in \mathbb{R}^{n}: A^{{ }^{k}} \otimes x \rightarrow \varepsilon$, as $k \rightarrow \infty$. From Eqs. 16 and 17 it follows that $x_{\mathrm{e}}=\bigoplus_{t=1}^{n} A^{\otimes^{n-t}} \otimes B \otimes u_{\mathrm{e}}$. Therefore, there exists a finite integer $k(x) \geq n$ such that $\phi\left(k ; x, u_{\mathrm{e}}\right)=x_{\mathrm{e}}$ for all $k \geq k(x)$, i.e. convergence towards the equilibrium $x_{\mathrm{e}}$ is achieved in finite number of steps. In fact, we can even determine an upper bound for $k(x)$. Indeed, since $A_{i j}<0$ for all $i$, $j$, then if $\ell \geq p n$ for some integers $\ell$ and $p$, it follows that $\left(A^{\otimes^{\ell}}\right)_{i j}$ is either equal to $\varepsilon$ or it is the weight of a path of length $\ell$ that contains at least $p$ cycles (see Baccelli et al. 1992, Chapter 2 for appropriate definitions for path and cycle, and for an interpretation of the MPA power $A^{{ }^{k}}$ in terms of graphs). Note that for any cycle the weight of the cycle is less than $\lambda_{\max }-\rho<0$. Since $\left(A^{{ }^{k}} \otimes x\right)_{i}=\max _{j}\left\{\left(A^{{ }{ }^{k}}\right)_{i j}+x_{j}\right\}$, it follows that by choosing ${ }^{6} p=\left\lfloor\max _{i, j} \frac{\left(x_{\mathrm{e}}\right)_{i}-x_{j}}{\lambda_{\max }-\rho}\right\rfloor$, we have $A^{{ }^{p n}} \otimes x \leq x_{\mathrm{e}}$. Furthermore, since $A_{i j}<0$ for all $i, j$, we have $A^{{ }^{p n+l}} \otimes x \leq x_{\mathrm{e}}$ for all $l \in \mathbb{N}$. Therefore, $p n$ is an upper bound on $k(x)$.

It now remains to prove that $X_{\mathrm{e}}=\left\{x_{\mathrm{e}}\right\}$ is stable. Note that $x_{\mathrm{e}}=A^{\otimes^{k}} \otimes x_{\mathrm{e}} \oplus$ $\left(\bigoplus_{t=1}^{k} A^{{ }^{k-t}} \otimes B \otimes u_{\mathrm{e}}\right)$ for all $k \geq 1$. Since we assume that $x_{\mathrm{e}}$ is finite, it follows that the matrix [ $A^{{ }^{k}} \bigoplus_{t=1}^{k} A^{{ }^{k-t}} \otimes B$ ] is row-finite for all $k$. Then, from Eq. 20 it follows that

$$
\left\|\phi\left(k ; x, u_{\mathrm{e}}\right)-x_{\mathrm{e}}\right\|_{\infty} \leq\left\|x-x_{\mathrm{e}}\right\|_{\infty} \forall x \in \mathbb{R}^{n}, \quad k \geq 0,
$$

i.e. the set $X_{\mathrm{e}}=\left\{x_{\mathrm{e}}\right\}$ is stable for Eq. 19 (here we have $\delta=\theta$ for Definition 2.3).

3.2 Maximal invariant set $\mathcal{O}_{\infty}$

We recall that by Assumptions A1-A2 we have $A<0$ and $H \geq 0$. We define the input-state admissible set associated with the closed-loop system 19 subject to the constraints 15

$$
\mathcal{O}_{0}:=\left\{x \in \mathbb{R}^{n}: H x+G u_{\mathrm{e}} \leq h\right\}
$$

We want to compute the maximal positively invariant set contained in the input-state admissible set $\mathcal{O}_{0}$ corresponding to the closed-loop system 19. Therefore, we define recursively the sets

$$
\mathcal{O}_{k}:=\left\{x \in \mathcal{O}_{0}: f\left(x, u_{\mathrm{e}}\right) \in \mathcal{O}_{k-1}\right\},
$$

for all $k \geq 1$. From the definition of the set $\mathcal{O}_{k}$ and using induction it follows that $\mathcal{O}_{k} \subseteq \mathcal{O}_{k-1}$ for all $k \geq 1$. Indeed, for $k=1$ the inclusion is obvious. Now let us assume that $\mathcal{O}_{k} \subseteq \mathcal{O}_{k-1}$ and prove that this implies that $\mathcal{O}_{k+1} \subseteq \mathcal{O}_{k}$. Using the definition of the set $\mathcal{O}_{k+1}$ and the induction hypothesis it follows that $\mathcal{O}_{k+1}=\left\{x \in \mathcal{O}_{0}: f\left(x, u_{\mathrm{e}}\right) \in\right.$ $\left.\mathcal{O}_{k}\right\} \subseteq\left\{x \in \mathcal{O}_{0}: f\left(x, u_{\mathrm{e}}\right) \in \mathcal{O}_{k-1}\right\}=\mathcal{O}_{k}$. Therefore, the limit of $\mathcal{O}_{k}$ exists and we have

$$
\mathcal{O}_{\infty}:=\lim _{k \rightarrow \infty} \mathcal{O}_{k}=\bigcap_{k \geq 0} \mathcal{O}_{k}
$$

\footnotetext{
${ }^{6}\lfloor x\rfloor$ denotes the largest integer less or equal to $x$.

Springer
} 
By induction we can prove that $x_{\mathrm{e}} \in \mathcal{O}_{k}$ for all $k \geq 0$ and therefore $x_{\mathrm{e}} \in \mathcal{O}_{\infty}$, i.e. $\mathcal{O}_{\infty}$ is non-empty.

Proposition 3.2 Suppose that Assumption A2 holds. Then, the sets $\mathcal{O}_{k}$ are polyhedra of the form

$$
\mathcal{O}_{k}=\left\{x \in \mathbb{R}^{n}: H_{k} x \leq h_{k}\right\},
$$

with $H_{k} \geq 0$.

Proof For $k=0$ the statement holds according to Assumption A2. Let us assume that $\mathcal{O}_{k-1}=\left\{x \in \mathbb{R}^{n}: H_{k-1} x \leq h_{k-1}\right\}$ with $H_{k-1} \geq 0$ and prove that $\mathcal{O}_{k}$ has a similar form. Since $A \otimes x \oplus B \otimes u_{\mathrm{e}}$ is a max expression in $x$ and $H_{k-1} \geq 0$, it follows that the inequality $H_{k-1} f\left(x, u_{\mathrm{e}}\right)=H_{k-1} \cdot\left(A \otimes x \oplus B \otimes u_{\mathrm{e}}\right) \leq h_{k-1}$ can be rewritten in the form $\bar{H}_{k} x \leq \bar{h}_{k}$ with $\bar{H}_{k} \geq 0$. So if we define $H_{k}=\left[\bar{H}_{k-1}^{T} \bar{H}_{k}^{T}\right]^{T}$ and $h_{k}=\left[h_{k-1}^{T} \bar{h}_{k}^{T}\right]^{T}$, then $H_{k} \geq 0$ and $\mathcal{O}_{k}$ can be written as Eq. 24 .

From the previous lemma it is clear that the set $\mathcal{O}_{\infty}$ is convex (it is a countable intersection of polyhedral sets). We now derive conditions when $\mathcal{O}_{\infty}$ is a polyhedron. We first give a definition:

Definition 3.3 The set $\mathcal{O}_{\infty}$ is finitely determined if there exists a finite positive integer $\tau$ such that $\mathcal{O}_{\infty}=\mathcal{O}_{\tau}$.

\section{Proposition 3.4}

(i) If there exists a finite positive integer $\tau$ such that $\mathcal{O}_{\tau}=\mathcal{O}_{\tau+1}$, then $\mathcal{O}_{\infty}$ is finitely determined and it is a polyhedral set.

(ii) The set $\mathcal{O}_{\infty}$ is the maximal positively invariant set for Eq. 19 contained in $\mathcal{O}_{0}$.

Proof

(i) Let us assume that there exists a finite positive integer $\tau$ such that $\mathcal{O}_{\tau}=$ $\mathcal{O}_{\tau+1}$. It is obvious that $\mathcal{O}_{\tau+2} \subseteq \mathcal{O}_{\tau+1}$. Moreover, for any $x \in \mathcal{O}_{\tau+1}$ it follows that $f\left(x, u_{\mathrm{e}}\right) \in \mathcal{O}_{\tau}=\mathcal{O}_{\tau+1}$, i.e. $x \in \mathcal{O}_{\tau+2}$. In conclusion, $\mathcal{O}_{\tau+1} \subseteq \mathcal{O}_{\tau+2}$ and thus $\mathcal{O}_{\tau+2}=\mathcal{O}_{\tau+1}=\mathcal{O}_{\tau}$. Iterating this procedure and using Eq. 23 we conclude that $\mathcal{O}_{\infty}=\mathcal{O}_{\tau}$. Since $\mathcal{O}_{\tau}$ is a polyhedron, it follows that $\mathcal{O}_{\infty}$ is also a polyhedral set.

(ii) Let $T \subseteq \mathcal{O}_{0}$ be a positively invariant set for Eq. 19 and let $x \in T$. Then from the definition of a positively invariant set we have $H_{0} f\left(x, u_{\mathrm{e}}\right) \leq h_{0}$. This implies that $x \in \mathcal{O}_{1}$ (according to the recursion Eq. 22). Therefore, $T \subseteq \mathcal{O}_{1}$. By iterating this procedure we obtain that $T \subseteq \mathcal{O}_{k}$ for all $k \geq 0$. In conclusion, for any positively invariant set $T$ for Eq. 19 it follows that $T \subseteq \mathcal{O}_{\infty}$ and thus $\mathcal{O}_{\infty}$ is maximal.

From Proposition 3.4 it follows that if $\mathcal{O}_{\infty}$ is finitely determined, then $\mathcal{O}_{\infty}$ is a polyhedron of the form $\mathcal{O}_{\infty}=\left\{x \in \mathbb{R}^{n}: H_{\infty} x \leq h_{\infty}\right\}$ with $H_{\infty} \geq 0$. Now, we give sufficient conditions under which the set $\mathcal{O}_{\infty}$ is finitely determined. Note that the recursive relation Eq. 22 can be written equivalently as

$$
\mathcal{O}_{k}=\left\{x \in \mathcal{O}_{k-1}: H \phi\left(k ; x, u_{\mathrm{e}}\right)+G u_{\mathrm{e}} \leq h\right\},
$$


where $\phi\left(k ; x, u_{\mathrm{e}}\right)$ can be written explicitly as $\phi\left(k ; x, u_{\mathrm{e}}\right)=A^{\otimes^{k}} \otimes x \oplus A^{\otimes^{k-1}} \otimes B \otimes$ $u_{\mathrm{e}} \oplus \cdots \oplus B \otimes u_{\mathrm{e}}$.

Theorem 3.5 Suppose that there exists a finite positive integer $\tau_{0}$ and a vector a $\in \mathbb{R}^{n}$ such that $\mathcal{O}_{\tau_{0}} \subseteq\left\{x \in \mathbb{R}^{n}: x \leq a\right\}$, and that Assumption A1 holds. Then $\mathcal{O}_{\infty}$ is finitely determined.

Proof Since $A_{i j}<0$ for all $i, j$ (according to Assumption A1), it follows that for all $x \in \mathbb{R}^{n}: A^{{ }^{k}} \otimes x \rightarrow \varepsilon$ as $k \rightarrow \infty$. Moreover, for any $b \in \mathbb{R}^{n}$ we have $b \oplus A \otimes b \oplus$ $\cdots \oplus A^{\otimes^{k+n}} \otimes b=A^{*} \otimes b$ for all $k \geq 0$. Since $x_{\mathrm{e}}=A^{*} \otimes B \otimes u_{\mathrm{e}}$ is finite, there exists a $\tau \geq \max \left\{n, \tau_{0}\right\}$ such that $A^{\otimes^{k}} \otimes a \leq x_{\mathrm{e}}$ for all $k \geq \tau$. We now have to show that $\mathcal{O}_{\tau}=\mathcal{O}_{\tau+1}$. Since $\mathcal{O}_{\tau+1} \subseteq \mathcal{O}_{\tau}$, to complete the proof we now show that the other inclusion is also valid, i.e. $\mathcal{O}_{\tau} \subseteq \mathcal{O}_{\tau+1}$.

Let $x \in \mathcal{O}_{\tau} \subseteq \mathcal{O}_{\tau_{0}} \subseteq\left\{x \in \mathbb{R}^{n}: x \leq a\right\}$. Then by Eq. 1 we have $A^{\otimes^{\tau+1}} \otimes x \leq$ $A^{\otimes^{\tau+1}} \otimes a \leq x_{\mathrm{e}}$. It follows that: $H \cdot\left(A^{\otimes^{\tau+1}} \otimes x \oplus A^{\otimes^{\tau}} \otimes B \otimes u_{\mathrm{e}} \oplus \cdots \oplus B \otimes u_{\mathrm{e}}\right)=$ $H \cdot\left(A^{\otimes^{\tau+1}} \otimes x \oplus A^{*} \otimes B \otimes u_{\mathrm{e}}\right)=H x_{\mathrm{e}} \leq h-G u_{\mathrm{e}}$, i.e. $x \in \mathcal{O}_{\tau+1}$ and thus $\mathcal{O}_{\tau} \subseteq \mathcal{O}_{\tau+1}$.

Remark 3.6 It is often the case that the set $\mathcal{O}_{0}$ can be written as $\mathcal{O}_{0}=\left\{x \in \mathbb{R}^{n}\right.$ : $x_{i} \leq a_{i}^{0}$, for $\left.i \in \mathbb{N}_{[1, n]}\right\}$, where $a_{i}^{0}$ is either a finite number or $+\infty$ (when there are no restrictions on $x_{i}$ ). Then, we can prove that all the sets $\mathcal{O}_{k}$ can be written in a similar form $\mathcal{O}_{k}=\left\{x \in \mathbb{R}^{n}: x_{i} \leq a_{i}^{k}\right.$, for $\left.i \in \mathbb{N}_{[1, n]}\right\}$, where $a_{i}^{k}$ is either a finite number or $+\infty$ (so every $\mathcal{O}_{k}$ is described by at most $n$ inequalities).

We prove this by induction. For $k=0$ this statement is true. Let us assume that $\mathcal{O}_{k}=\left\{x \in \mathbb{R}^{n}: x_{i} \leq a_{i}^{k}\right.$, for $\left.i \in \mathbb{N}_{[1, n]}\right\}$ and prove that $\mathcal{O}_{k+1}$ has a similar form. We denote with $a^{k}=\left[a_{1}^{k} \cdots a_{n}^{k}\right]^{T}$. From the recursive relation (Eq. 22) we have

$$
\begin{aligned}
\mathcal{O}_{k+1} & =\left\{x \in \mathbb{R}^{n}: x \leq a^{k}, A \otimes x \leq a^{k}\right\} \\
& =\left\{x \in \mathbb{R}^{n}: x \leq a^{k}, x \leq\left(-A^{T}\right) \otimes^{\prime} a^{k}\right\}=\left\{x \in \mathbb{R}^{n}: x \leq a^{k+1}\right\},
\end{aligned}
$$

where $a^{k+1}=\min \left\{a^{k},\left(-A^{T}\right) \otimes^{\prime} a^{k}\right\}$ (recall that the operator $\otimes^{\prime}$ is defined in Section 2.1). We conclude that $\mathcal{O}_{\infty}$ is described by at most $n$ inequalities and in fact $\mathcal{O}_{\infty}=$ $\left\{x \in \mathbb{R}^{n}: x \leq a^{\infty}\right\}$ where $a_{i}^{\infty}$ is either in $\mathbb{R}$ or equal to $+\infty$ for any $i \in \mathbb{N}_{[1, n]}$.

Note that the results obtained in this section concerning the maximal positively invariant set $\mathcal{O}_{\infty}$ for the MPL system 19 are similar to the ones obtained in (Gilbert and Tan 1991) for conventional, time-driven linear systems.

\section{Stable model predictive control for MPL systems}

The main advantage of MPC is that it can accommodate constraints on inputs and states. In this section it is assumed that the maximal positively invariant set $\mathcal{O}_{\infty}$ is available and that it is a polyhedron, i.e. $\mathcal{O}_{\infty}=\left\{x \in \mathbb{R}^{n}: H_{\infty} x \leq h_{\infty}\right\}$ with $H_{\infty} \geq 0$ (according to Section 3.2). 
4.1 State regulation

We first give a lemma that will be used in the sequel:

\section{Proposition 4.1}

(i) Let $\mathcal{X}=\left\{x \in \mathbb{R}^{n}: P x \leq q\right\}$, where $P \geq 0$, be a non-empty set and let $x_{0} \in \mathbb{R}^{n}$. Then $d_{\infty}\left(x_{0}, \mathcal{X}\right)=\min _{x \in \mathcal{X}} \max \left\{\left\|x_{0}-x\right\|_{\oplus}, 0\right\}$.

(ii) In particular if $\mathcal{X}=\left\{x \in \mathbb{R}^{n}: x \leq a\right\}$, then $d_{\infty}\left(x_{0}, \mathcal{X}\right)=\max \left\{\left\|x_{0}-a\right\|_{\oplus}, 0\right\}$.

\section{Proof}

(i) Note that since $x_{0}$ is finite and since the points of $\mathcal{X}$ are also finite and $\mathcal{X}$ is non-empty and closed, the distance $d_{\infty}\left(x_{0}, \mathcal{X}\right)$ is defined and finite.

First we consider the case where $x_{0} \in \mathcal{X}$. Then we have $d_{\infty}\left(x_{0}, \mathcal{X}\right)=0$ and $\min _{x \in \mathcal{X}}\left\|x_{0}-x\right\|_{\oplus} \leq 0$ (note that for $x=x_{0}$ we have $\left\|x_{0}-x\right\|_{\oplus}=0$ which implies that the minimum - or better, infimum, in this case - will be less than or equal to 0 ). This implies that the statement of part (i) of the proposition holds if $x_{0} \in \mathcal{X}$.

From now on we consider the case when $x_{0} \notin \mathcal{X}$.

Clearly, $d_{\infty}\left(x_{0}, \mathcal{X}\right)=\min _{x \in \mathcal{X}}\left\|x_{0}-x\right\|_{\infty}>0$ if $x_{0} \notin \mathcal{X}$. Let us now prove that also $\min _{x \in \mathcal{X}}\left\|x_{0}-x\right\|_{\oplus}>0$ if $x_{0} \notin \mathcal{X}$. We do this by contradiction. Suppose that there exists an $\tilde{x} \in \mathcal{X}$ such that $\left\|x_{0}-\tilde{x}\right\|_{\oplus} \leq 0$. Then we have $\max _{i}\left\{\left(x_{0}\right)_{i}-\tilde{x}_{i}\right\} \leq$ 0 and thus $\left(x_{0}\right)_{i}-\tilde{x}_{i} \leq 0$ for all $i$, which implies that $x_{0} \leq \tilde{x}$. Since $P \geq 0$, this results in $P x_{0} \leq P \tilde{x} \leq q$. So $x_{0} \in \mathcal{X}$, which is in contradiction with the fact that $x_{0} \notin \mathcal{X}$. As a consequence, we have $\left\|x_{0}-x\right\|_{\oplus}>0$ for all $x \in \mathcal{X}$. Since $\inf _{x \in \mathcal{X}}\left\|x_{0}-x\right\|_{\oplus}$ can be recast $^{7}$ as a linear programming problem that is feasible and for which the objective function is (strictly) bounded from below by 0 , the infimum is attained, which implies that the minimum $\min _{x \in \mathcal{X}}\left\|x_{0}-x\right\|_{\oplus}$ exists and satisfies $\min _{x \in \mathcal{X}}\left\|x_{0}-x\right\|_{\oplus}>0$.

Let $x \in \mathbb{R}^{n}$. From the definition of $\left\|x_{0}-x\right\|_{\oplus}$ and $\left\|x_{0}-x\right\|_{\infty}$, it directly follows that $\left\|x_{0}-x\right\|_{\oplus} \leq\left\|x_{0}-x\right\|_{\infty}$ for any $x \in \mathbb{R}^{n}$. This implies that $\min _{x \in \mathcal{X}} \| x_{0}-$ $x\left\|_{\oplus} \leq \min _{x \in \mathcal{X}}\right\| x_{0}-x \|_{\infty}$. So to complete the proof of part (i) we have to prove that we also have $\min _{x \in \mathcal{X}}\left\|x_{0}-x\right\|_{\oplus} \geq \min _{x \in \mathcal{X}}\left\|x_{0}-x\right\|_{\infty}$.

Let $x^{\dagger}$ be a point of $\mathcal{X}$ for which $\left\|x_{0}-x^{\dagger}\right\|_{\oplus}=\min _{x \in \mathcal{X}}\left\|x_{0}-x\right\|_{\oplus}$. For $\| x_{0}-$ $x^{\dagger} \|_{\infty}$ we now distinguish between two cases:

Case A: There exists an index $i \in \mathbb{N}_{[1, n]}$ such that $\left\|x_{0}-x^{\dagger}\right\|_{\infty}=\left(x_{0}\right)_{i}-x_{i}^{\dagger}$. Then we have $\left(x_{0}\right)_{j}-x_{j}^{\dagger} \leq\left(x_{0}\right)_{i}-x_{i}^{\dagger}$ for all $j \in \mathbb{N}_{[1, n]}$. Hence, $\| x_{0}-$ $x^{\dagger}\left\|_{\oplus}=\left(x_{0}\right)_{i}-x_{i}^{\dagger}=\right\| x_{0}-x^{\dagger} \|_{\infty}$. So in this case, $\left\|x_{0}-x^{\dagger}\right\|_{\oplus}=\left\|x_{0}-x^{\dagger}\right\|_{\infty}$.

Case B: We have $\left\|x_{0}-x^{\dagger}\right\|_{\infty}>\left(x_{0}\right)_{j}-x_{j}^{\dagger}$ for all $j \in \mathbb{N}_{[1, n]}$.

\footnotetext{
${ }^{7}$ By introducing a dummy variable $t$ such that $t \geqslant\left\|x_{0}-x\right\|_{\oplus}$ or equivalently $t \geq\left(x_{0}\right)_{i}-x_{i}$ for all $i$, and then minimizing $t$ subject to these constraints and to $P x \leq q$, we obtain a linear programming problem. It is easy to verify that for the optimal solution $\left(t_{\mathrm{opt}}, x_{\mathrm{opt}}\right)$ of this linear programming problem we have $t_{\mathrm{opt}}=\left\|x_{0}-x_{\mathrm{opt}}\right\|_{\oplus}$.
} 
Now we will prove that this case can be transformed and reduced to Case $\mathbf{A}$. If $\left\|x_{0}-x^{\dagger}\right\|_{\infty}>\left(x_{0}\right)_{j}-x_{j}^{\dagger}$ for all $j \in \mathbb{N}_{[1, n]}$, then there should exist an index $i \in \mathbb{N}_{[1, n]}$ such that $\left\|x_{0}-x^{\dagger}\right\|_{\infty}=x_{i}^{\dagger}-\left(x_{0}\right)_{i}$. Since $\left\|x_{0}-x^{\dagger}\right\|_{\infty}>0$, we then have $x_{i}^{\dagger}>\left(x_{0}\right)_{i}$. This implies that the set $\mathcal{I}=\left\{i \in \mathbb{N}_{[1, n]}: x_{i}^{\dagger}>\left(x_{0}\right)_{i}\right\}$ is non-empty. If we now define $x_{\text {feas }} \in \mathbb{R}^{n}$ such that $\left(x_{\text {feas }}\right)_{i}=\left(x_{0}\right)_{i}$ if $i \in \mathcal{I}$, and $\left(x_{\text {feas }}\right)_{i}=x_{i}^{\dagger}$ if $i \notin \mathcal{I}$, then we have $x_{\text {feas }} \leq x^{\dagger}$. Since $P \geq 0$, this implies that $P x_{\text {feas }} \leq P x^{\dagger} \leq q$ and thus $x_{\text {feas }} \in \mathcal{X}$. Moreover, we have

$$
\begin{aligned}
\left\|x_{0}-x^{\dagger}\right\|_{\oplus} & \left.\leq\left\|x_{0}-x_{\text {feas }}\right\|_{\oplus} \quad \text { (by the definition of } x^{\dagger} \text { and as } x_{\text {feas }} \in \mathcal{X}\right) \\
& =\max _{l \in \mathbb{N}_{[1, n]}}\left\{\left(x_{0}\right)_{l}-\left(x_{\text {feas }}\right)_{l}\right\} \\
& =\max \left\{\max _{i \in \mathcal{I}}\left\{\left(x_{0}\right)_{i}-\left(x_{\text {feas }}\right)_{i}\right\}, \max _{j \in \mathbb{N}_{[1, n]} \backslash \mathcal{I}}\left\{\left(x_{0}\right)_{j}-\left(x_{\text {feas }}\right)_{j}\right\}\right\} \\
& =\max \left\{0, \max _{j \in \mathbb{N}_{[1, n]} \backslash \mathcal{I}}\left\{\left(x_{0}\right)_{j}-\left(x_{\text {feas }}\right)_{j}\right\}\right\} \quad \text { (as }\left(x_{\text {feas }}\right)_{i}=\left(x_{0}\right)_{i} \\
& \leqslant\left\|x_{0}-x^{\dagger}\right\|_{\oplus} \quad\left(\text { as }\left\|x_{0}-x^{\dagger}\right\|_{\oplus}>0 \text { and by the definition of } \mathcal{I}\right) .
\end{aligned}
$$

So $\left\|x_{0}-x^{\dagger}\right\|_{\oplus}=\left\|x_{0}-x_{\text {feas }}\right\|_{\oplus}$, which means that also for $x_{\text {feas }}$ we have $\| x_{0}-$ $x_{\text {feas }}\left\|_{\oplus}=\min _{x \in \mathcal{X}}\right\| x_{0}-x \|_{\oplus}$. Now we show that if we redefine $x^{\dagger}$ to be equal to $x_{\text {feas }}$ then Case $\mathbf{A}$ holds. Indeed, we have

$$
\begin{aligned}
\left\|x_{0}-x_{\text {feas }}\right\|_{\infty} & =\max _{l \in \mathbb{N}_{[1, n]}}\left\{\max \left\{\left(x_{0}\right)_{l}-\left(x_{\text {feas }}\right)_{l},\left(x_{\text {feas }}\right)_{l}-\left(x_{0}\right)_{l}\right\}\right\} \\
& =\max \left\{\max _{i \in \mathcal{I}}\left\{\max \left\{\left(x_{0}\right)_{i}-\left(x_{\text {feas }}\right)_{i},\left(x_{\text {feas }}\right)_{i}-\left(x_{0}\right)_{i}\right\}\right\},\right. \\
& \left.\max _{i \in \mathbb{N}_{[1, n]} \backslash \mathcal{I}}\left\{\max \left\{\left(x_{0}\right)_{i}-\left(x_{\text {feas }}\right)_{i},\left(x_{\text {feas }}\right)_{i}-\left(x_{0}\right)_{i}\right\}\right\}\right\} \\
& =\max \left\{0, \max _{i \in \mathbb{N}_{[1, n]} \backslash \mathcal{I}}\left\{\left(x_{0}\right)_{i}-\left(x_{\text {feas }}\right)_{i}\right\}\right\}
\end{aligned}
$$

by the definition of $\mathcal{I}$ and since for $i \notin \mathcal{I}$ we have $\left(x_{\text {feas }}\right)_{i}=x_{i}^{\dagger} \leq\left(x_{0}\right)_{i}$. Since $\left\|x_{0}-x_{\text {feas }}\right\|_{\infty}>0$, it follows that $\left\|x_{0}-x_{\text {feas }}\right\|_{\infty}=\max _{i \in \mathbb{N}_{[1, n]} \backslash \mathcal{I}}\left\{\left(x_{0}\right)_{i}-\left(x_{\text {feas }}\right)_{i}\right\}$, i.e., $x_{\text {feas }}$ satisfies Case $\mathbf{A}$ above, and thus $\left\|x_{0}-x_{\text {feas }}\right\|_{\oplus}=\left\|x_{0}-x_{\text {feas }}\right\|_{\infty}$.

In conclusion, we can always find a point $x^{\dagger} \in \mathcal{X}$ for which $\left\|x_{0}-x^{\dagger}\right\|_{\oplus}=$ $\min _{x \in \mathcal{X}}\left\|x_{0}-x\right\|_{\oplus}=\left\|x_{0}-x^{\dagger}\right\|_{\infty}$. This implies that $\min _{x \in \mathcal{X}}\left\|x_{0}-x\right\|_{\oplus}=\| x_{0}-$ $x^{\dagger}\left\|_{\oplus}=\right\| x_{0}-x^{\dagger}\left\|_{\infty} \geq \min _{x \in \mathcal{X}}\right\| x_{0}-x \|_{\infty}$. Together with the reverse inequality obtained previously, this results in $\min _{x \in \mathcal{X}}\left\|x_{0}-x\right\|_{\oplus}=\min _{x \in \mathcal{X}}\left\|x_{0}-x\right\|_{\infty}$, which concludes the proof of part (i).

(ii) If $x_{0} \in \mathcal{X}$, then $d_{\infty}\left(x_{0}, \mathcal{X}\right)=0$ and also $\left\|x_{0}-a\right\|_{\oplus} \leq 0$, which means that the result holds in this case.

If $x_{0} \notin \mathcal{X}$ and $x \leq a$, then $d_{\infty}\left(x_{0}, \mathcal{X}\right)>0$ and $x_{0}-x \geqslant x_{0}-a$. So $\max _{i}\left\{\left(x_{0}\right)_{i}-\right.$ $\left.x_{i}\right\} \geqslant \max _{i}\left\{\left(x_{0}\right)_{i}-a_{i}\right\}$ and thus also $d_{\infty}\left(x_{0}, \mathcal{X}\right)=\min _{x \in \mathcal{X}} \max _{i}\left\{\left(x_{0}\right)_{i}-x_{i}\right\} \geqslant$ $\max _{i}\left\{\left(x_{0}\right)_{i}-a_{i}\right\}$. So $d_{\infty}\left(x_{0}, \mathcal{X}\right) \geqslant\left\|x_{0}-a\right\|_{\oplus}$. On the other hand, from part (i) of this proposition it follows that $0<d_{\infty}\left(x_{0}, \mathcal{X}\right)=\min _{x \in \mathcal{X}} \max _{i}\left\{\left(x_{0}\right)_{i}-x_{i}\right\} \leqslant$ $\left\|x_{0}-a\right\|_{\oplus}$. Hence, $d_{\infty}\left(x_{0}, \mathcal{X}\right)=\left\|x_{0}-a\right\|_{\oplus}$. 
The MPC strategy derived in this section uses $\mathcal{O}_{\infty}$ as the terminal set, where we recall that $\mathcal{O}_{\infty}$ is a polyhedron of the form $\mathcal{O}_{\infty}=\left\{x \in \mathbb{R}^{n}: H_{\infty} x \leq h_{\infty}\right\}$ with $H_{\infty} \geq 0$. For a given positively invariant set $X_{\mathrm{e}}$ for Eq. 19 such that $x_{\mathrm{e}} \in \bar{X}_{\mathrm{e}} \subseteq \mathcal{O}_{\infty}$, we define a continuous stage $\operatorname{cost} \ell(x, u)$ with the following properties:

P1: $\quad \ell(x, u)=0$ iff $x \in X_{\mathrm{e}}$ and $u=u_{\mathrm{e}}$.

P2: $\quad \ell(x, u) \geq \alpha\left(d_{\infty}\left(x, X_{\mathrm{e}}\right)+\left\|u-u_{\mathrm{e}}\right\|_{\infty}\right)$ for all $x$ and $u$, where $\alpha$ is a $\mathcal{K}$-function.

Some examples of such stage costs are

$$
\begin{aligned}
& \ell(x, u)=\left\|x-x_{\mathrm{e}}\right\|_{\infty}+\gamma\left\|u-u_{\mathrm{e}}\right\|_{\infty} \\
& \ell(x, u)=\max _{i \in \mathbb{N}_{[1, n]}}\left\{x_{i}-\left(x_{\mathrm{e}}\right)_{i}, 0\right\}+\gamma\left\|u-u_{\mathrm{e}}\right\|_{\infty} \\
& \ell(x, u)=d_{\infty}\left(x, \mathcal{O}_{\infty}\right)+\gamma\left\|u-u_{\mathrm{e}}\right\|_{\infty},
\end{aligned}
$$

where $\gamma>0$, i.e. it is a positive scalar. The stage cost (Eq. 26) corresponds to $X_{\mathrm{e}}=\left\{x_{\mathrm{e}}\right\}$, Eq. 27 corresponds to $X_{\mathrm{e}}=\left\{x: x \leq x_{\mathrm{e}}\right\}$ (according to Proposition 4.1), and Eq. 28 corresponds to $X_{\mathrm{e}}=\mathcal{O}_{\infty}$. Note that the first term in these stage costs penalizes the tardiness with respect to the boundary of the set $X_{\mathrm{e}}$ while the second term penalizes the deviation of the input from the equilibrium input $u_{\mathrm{e}}$. From Proposition 4.1 it follows that in these cases the $\mathcal{K}$-function $\alpha$ of Property $\mathbf{P 2}$ is the identity function, i.e. $\alpha(x)=x$.

We consider a prediction horizon $N \geq 1$. For the event pair $(k, x)$ (i.e. $x(k)=x)$ the following optimal control problem is considered:

$$
\mathbb{P}_{N}(x): \quad V_{N}^{0}(x):=\min _{\mathbf{u} \in \Pi_{N}(x)} V_{N}(x, \mathbf{u}),
$$

where the set of feasible input sequences is defined by ${ }^{8}$

$$
\Pi_{N}(x):=\left\{\mathbf{u}: H x_{i}+G u_{i} \leq h \forall i \in \mathbb{N}_{[1, N]}, x_{N} \in \mathcal{O}_{\infty}\right\},
$$

and the cost function is defined by $V_{N}: \mathbb{R}^{n} \times \mathbb{R}^{N m} \rightarrow \mathbb{R}$

$$
V_{N}(x, \mathbf{u})=\sum_{i=1}^{N} \ell\left(x_{i-1}, u_{i}\right)+V_{\mathrm{f}}\left(x_{N}\right),
$$

where the stage cost $\ell$ satisfies Properties P1-P2, and where $\mathbf{u}:=\left[u_{1}^{T} \cdots u_{N}^{T}\right]^{T}$ and $x_{i}:=\phi(i ; x, \mathbf{u})$. It follows that $x_{0}=x$. The terminal cost is determined as $V_{\mathrm{f}}: \mathcal{O}_{\infty} \rightarrow \mathbb{R}$

$$
V_{\mathrm{f}}\left(x_{N}\right):=\sum_{j=1}^{k\left(x_{N}\right)} \ell\left(x_{j}, u_{\mathrm{e}}\right),
$$

where $k\left(x_{N}\right)$ is finite and defined as in the proof of Theorem 3.1 and $x_{j}:=\phi\left(j ; x_{N}\right.$, $u_{\mathrm{e}}$ ). Typically $\mathcal{O}_{\infty} \subseteq\{x: x \leq a\}$ (see Remark 3.6) and then an upper bound on $k(x)$ is $k(a)$, where $k(a)$ can be determined as in the proof of Theorem 3.1. Note that for the stage cost Eq. 28 we always have a zero terminal cost since $V_{\mathrm{f}}(x)=0$ for all $x \in \mathcal{O}_{\infty}$.

\footnotetext{
${ }^{8}$ So $\Pi_{N}(x)$ is the set of input sequences for which starting from the initial state $x$ the constraints (15) are satisfied and for which the target set $\mathcal{O}_{\infty}$ is reached after $N$ steps.
} 
Let $X_{N}$ denote the set of finite initial states for which a feasible input sequence exists, i.e.

$$
X_{N}:=\left\{x \in \mathbb{R}^{n}: \Pi_{N}(x) \neq \emptyset\right\}
$$

The optimal control problem $\mathbb{P}_{N}(x)$ yields an optimal control sequence $\mathbf{u}^{0}(x)=$ $\left[\left(u_{1}^{0}(x)\right)^{T} \cdots\left(u_{N}^{0}(x)\right)^{T}\right]^{T}$ for all $x \in X_{N}$. The first control $u_{1}^{0}(x)$ is applied to the system (Eqs. 13 and 14) at step $k$ according to the receding horizon principle. This defines an implicit MPC law $\kappa^{\mathrm{MPC}}(x):=u_{1}^{0}(x)$. The next theorem shows that the closed-loop system obtained from applying the MPC law $\kappa^{\mathrm{MPC}}$ to Eqs. 13 and 14 enjoys some stabilizing properties.

Theorem 4.2 Suppose that $X_{\mathrm{e}}$ lies in the interior of the set $X_{N}$ and that Assumption A1 holds. Then,

(i) the set $X_{\mathrm{e}}$ is asymptotically stable for the closed-loop system

$$
x(k)=A \otimes x(k-1) \oplus B \otimes \kappa^{\mathrm{MPC}}(x(k-1))
$$

with a region of attraction $X_{N}$, and

(ii) if there exists an $a \in \mathbb{R}^{n}$ such that $X_{\mathrm{e}} \subseteq\left\{x \in \mathbb{R}^{n}: x \leq a\right\}$, then for each $x \in X_{N}$ the closed-loop state trajectory of the system 30 is bounded.

Proof

(i) Consider the function $V_{N}^{0}: X_{N} \rightarrow \mathbb{R}$ defined by Eq. 29. We will show that $V_{N}^{0}$ satisfies the conditions from Theorem 2.5.

Let us show that $X_{N}$ is positively invariant for the system (Eq. 30). Let $x \in X_{N}$, then there exists an optimal control sequence $\mathbf{u}^{0}(x) \in \Pi_{N}(x)$. Moreover, let $\mathbf{x}^{0}=\left[x^{T}\left(x_{1}^{0}\right)^{T} \cdots\left(x_{N}^{0}\right)^{T}\right]^{T}$ be the corresponding optimal state trajectory. The MPC input $\kappa^{\mathrm{MPC}}(x)$ steers the system from the state $x$ to the successor state $x_{1}^{0}=f\left(x, \kappa^{\mathrm{MPC}}(x)\right)$. Since $x_{N}^{0} \in \mathcal{O}_{\infty}$, we have $f\left(x_{N}^{0}, u_{\mathrm{e}}\right) \in \mathcal{O}_{\infty}$. Furthermore, the feasible control sequence $\left[\left(u_{2}^{0}(x)\right)^{T} \cdots\left(u_{N}^{0}(x)\right)^{T}\right]^{T}$ steers the system from the state $x_{1}^{0}$ to $x_{N}^{0} \in \mathcal{O}_{\infty}$. It follows that at the next step a feasible input sequence is given by $\mathbf{u}^{\mathrm{f}}=\left[\left(u_{2}^{0}(x)\right)^{T} \cdots\left(u_{N}^{0}(x)\right)^{T} u_{\mathrm{e}}^{T}\right]^{T}$, i.e. $\mathbf{u}^{\mathrm{f}} \in \Pi_{N}\left(f\left(x, \kappa^{\mathrm{MPC}}(x)\right)\right)$. We conclude that $f\left(x, \kappa^{\mathrm{MPC}}(x)\right) \in X_{N}$ and thus $X_{N}$ is a positively invariant set for Eq. 30. As a consequence, for any initial state $x \in X_{N}$ we can guarantee feasibility of the MPL-MPC optimization problem 29 at each step.

Using the properties of a multi-parametric convex program (see e.g. Necoara 2006), the Properties P1-P2 of the stage cost, convexity of the function $f$, and linearity of the constraints we can see that the first two conditions from Theorem 2.5 are satisfied by $V_{N}^{0}$. In particular, continuity of $V_{N}^{0}$ for the stage cost 26 follows from Eq. 20, while for the stage costs 27 and 28 continuity of $V_{N}^{0}$ follows from multi-parametric linear programming arguments (Necoara 2006, Section 2.3.1). It remains to prove the third condition. Due to the special form of the chosen feasible input sequence $\mathbf{u}^{\mathrm{f}}$, the input sequence $\left[\left(u_{2}^{0}(x)\right)^{T} \cdots\left(u_{N}^{0}(x)\right)^{T}\right]^{T}$ steers the system from the state $x_{1}^{0}$ to $x_{N}^{0} \in \mathcal{O}_{\infty}$ and then 
to $f\left(x_{N}^{0}, u_{\mathrm{e}}\right) \in \mathcal{O}_{\infty}$. Moreover, the terminal cost $V_{\mathrm{f}}$ is a finite sum of the stage costs $\ell$ over a horizon $k\left(x_{N}^{0}\right)$ corresponding to the input $u_{\mathrm{e}}$ and thus

$$
\begin{aligned}
& V_{\mathrm{f}}\left(x_{N}^{0}\right)=\sum_{j=0}^{k\left(x_{N}^{0}\right)} \ell\left(x_{N+j}^{0}, u_{\mathrm{e}}\right) \\
& V_{N}^{0}(x)=\sum_{i=1}^{N} \ell\left(x_{i-1}^{0}, u_{i}^{0}(x)\right)+V_{\mathrm{f}}\left(x_{N}^{0}\right) \\
& V_{N}\left(f\left(x, \kappa^{\mathrm{MPC}}(x)\right), \mathbf{u}^{\mathrm{f}}\right)=\sum_{i=2}^{N} \ell\left(x_{i-1}^{0}, u_{i}^{0}(x)\right)+V_{\mathrm{f}}\left(x_{N}^{0}\right),
\end{aligned}
$$

where $x_{N+j}^{0}=\phi\left(j ; x_{N}^{0}, u_{\mathrm{e}}\right)$ and $x_{0}^{0}:=x$. Then it follows that

$$
\begin{aligned}
V_{N}^{0}\left(f\left(x, \kappa^{\mathrm{MPC}}(x)\right)\right)-V_{N}^{0}(x) & \leq V_{N}\left(f\left(x, \kappa^{\mathrm{MPC}}(x)\right), \mathbf{u}^{\mathrm{f}}\right)-V_{N}^{0}(x) \\
& =-\ell\left(x, u_{1}^{0}(x)\right) \leq-\alpha\left(d_{\infty}\left(x, X_{\mathrm{e}}\right)\right)
\end{aligned}
$$

and according to Property $\mathbf{P 2}$ of the stage cost, we obtain that the conditions from Theorem 2.5 are satisfied. Therefore, $X_{\mathrm{e}}$ is asymptotically stable for Eq. 30 with a region of attraction $X_{N}$.

(ii) For any finite initial state $x \in X_{N}$, from (31) it follows that the sequence $\left\{V_{N}^{0}\left(\phi\left(k ; x, \kappa^{\mathrm{MPC}}\right)\right)\right\}_{k \geq 0}$ is non-increasing and bounded from below and thus convergent. Moreover, $\ell\left(\phi\left(k ; x, \kappa^{\mathrm{MPC}}\right), \kappa^{\mathrm{MPC}}\left(\phi\left(k ; x, \kappa^{\mathrm{MPC}}\right)\right)\right) \leq V_{N}^{0}$ $\left(\phi\left(k ; x, \kappa^{\mathrm{MPC}}\right)\right)-V_{N}^{0}\left(\phi\left(k+1 ; x, \kappa^{\mathrm{MPC}}\right)\right)$. Therefore, $\lim _{k \rightarrow \infty} \ell\left(\phi\left(k ; x, \kappa^{\overline{\mathrm{MPC}}}\right)\right.$, $\left.\kappa^{\mathrm{MPC}}\left(\phi\left(k ; x, \kappa^{\mathrm{MPC}}\right)\right)\right)=0$. Using continuity arguments and Properties P1-P2 of the stage cost it follows that

$$
\begin{aligned}
& \lim _{k \rightarrow \infty} \kappa^{\mathrm{MPC}}\left(\phi\left(k ; x, \kappa^{\mathrm{MPC}}\right)\right)=u_{\mathrm{e}} \\
& \lim _{k \rightarrow \infty} d_{\infty}\left(\phi\left(k ; x, \kappa^{\mathrm{MPC}}\right), X_{\mathrm{e}}\right)=0 .
\end{aligned}
$$

Since the initial state $x$ is taken to be finite and since the system is controllable and observable (according to Assumption A1), there does not exist a finite $k_{0}$ such that either $\phi\left(k_{0} ; x, \kappa^{\mathrm{MPC}}\right)$ or $\kappa^{\mathrm{MPC}}\left(\phi\left(k_{0} ; x, \kappa^{\mathrm{MPC}}\right)\right)$ or $y\left(k_{0}\right)=C \otimes$ $\phi\left(k_{0} ; x, \kappa^{\mathrm{MPC}}\right)$ are equal to $\varepsilon$. If the set $X_{\mathrm{e}}$ is bounded (e.g. $X_{\mathrm{e}}=\left\{x_{\mathrm{e}}\right\}$ in Eq. 26), then $\left\|\phi\left(k ; x, \kappa^{\mathrm{MPC}}\right)-x_{\mathrm{e}}\right\|_{\infty}$ is also bounded for all $k \geq 0$ (this follows from the triangle inequality for norms) and thus the buffer levels remain bounded.

If $X_{\mathrm{e}}$ is not bounded, then from Eq. 32 we conclude that for any finite initial state $x \in X_{N}$ there exists a finite lower bound $\underline{u}(x)$ such that $\kappa^{\mathrm{MPC}}\left(\phi\left(k ; x, \kappa^{\mathrm{MPC}}\right)\right) \geq \underline{u}(x)$ for all $k \geq 0$. From the monotonicity property of the max operator (Eq. 1) it follows that there exists a finite lower bound ${ }^{9}$ on the corresponding state trajectory $\phi\left(k ; x, \kappa^{\mathrm{MPC}}\right) \geq m(x)$ for all $k \geq 0$. Since $X_{\mathrm{e}} \subseteq$ $\left\{x \in \mathbb{R}^{n}: x \leq a\right\}$, it follows that the set $X_{\mathrm{e}} \cap\{z: z \geq m(x)\}$ is bounded and then using the same arguments as before we conclude that $\left\|\phi\left(k ; x, \kappa^{\mathrm{MPC}}\right)-x_{\mathrm{e}}\right\|_{\infty}$ is also bounded for all $k \geq 0$.

\footnotetext{
${ }^{9}$ I.e. $m(x):=A^{*} \otimes x \oplus A^{*} \otimes B \otimes \underline{u}(x)$ which is a finite vector.
} 
Remark 4.3

(i) Since in the constraints (Eq. 15) we have $H \geq 0$ (according to Assumption A2), and since for the terminal set $\mathcal{O}_{\infty}$ we have $H_{\infty} \geq 0$, using basic properties of the max operator it follows that $\Pi_{N}(x)$ is a polyhedron, i.e. it is described by linear inequalities: $\Pi_{N}(x)=\{\mathbf{u}: \mathcal{G} \mathbf{u} \leq \mathcal{H} x+g\}$. Furthermore, the set of initial states $X_{N}$ is also a polyhedron since $X_{N}$ is the projection of the polyhedral set $\{(x, \mathbf{u}): \mathcal{G} \mathbf{u}-\mathcal{H} x \leq g\}$ onto $\mathbb{R}^{n}$. For the stage costs 27 or 28 , using Proposition 4.1 , the previous discussion and including extra variables, it follows that the optimization problem (29) can be recast as a linear program (cf. Footnote 7). For the stage cost (26) the optimization problem 29 can be recast as a mixedinteger linear program, since in this case we also get that constraints that state that a maximum of linear expressions should be larger than or equal to some dummy variables. Such a constraint is not linear. However, by introducing additional binary variables such a constraint can be recast as a system of linear inequalities (Heemels et al. 2001). The overall problem then results in a mixedinteger linear programming problem.

(ii) If $X_{\mathrm{e}} \subset \operatorname{int}\left(\mathcal{O}_{\infty}\right)$, then from Eq. 33 it follows that the trajectory enters the terminal set $\mathcal{O}_{\infty}$ in a finite number of steps. Inside $\mathcal{O}_{\infty}$ we can use the feasible controller $u_{\mathrm{e}}$ (since $\mathcal{O}_{\infty}$ is a positively invariant set for the system 19) and so we can steer the trajectory towards the equilibrium $x_{\mathrm{e}}$ in finite number of steps as well (see Theorem 3.1). In conclusion, using such a dual-mode approach (see also Mayne et al. 2000), we can guarantee that for any finite initial state $x \in X_{N}$, the trajectory reaches the steady state in finite number of steps.

(iii) Note that by increasing the prediction horizon $N$, the region of attraction increases as well, i.e. for $N_{1}<N_{2}$ it follows that $X_{N_{1}} \subseteq X_{N_{2}}$. Indeed, let $x \in X_{N_{1}}$ then there exists a feasible $\mathbf{u}=\left[u_{1}^{T} \cdots u_{N_{1}}^{T}\right]^{T} \in \Pi_{N_{1}}(x)$ and we can construct $\mathbf{u}^{\mathrm{f}}=[u_{1}^{T} \cdots u_{N_{1}}^{T} \underbrace{u_{\mathrm{e}}^{T} \cdots u_{\mathrm{e}}^{T}}_{N_{2}-N_{1} \text { times }}]^{T} \in \Pi_{N_{2}}(x)$, i.e. $x \in X_{N_{2}}$.

\subsection{Output regulation}

For a given set $Y_{\mathrm{e}}$ such that $y_{\mathrm{e}}:=C \otimes x_{\mathrm{e}} \in Y_{\mathrm{e}}$, we define a continuous stage cost $\ell(x, u)$ with the following properties:

P1: $\quad \ell(x, u)=0$ iff $C \otimes x \in Y_{\mathrm{e}}$ and $u=u_{\mathrm{e}}$.

P2': $\quad \ell(x, u) \geq \alpha\left(d_{\infty}\left(y, Y_{\mathrm{e}}\right)+\left\|u-u_{\mathrm{e}}\right\|_{\infty}\right)$ for all $y=C \otimes x$ and $u$, where $\alpha$ is a $\mathcal{K}$-function.

Examples of such stage costs are [see De Schutter and van den Boom (2001) for more examples]

$$
\begin{aligned}
& \ell(x, u)=\left\|y-y_{\mathrm{e}}\right\|_{\infty}+\gamma\left\|u-u_{\mathrm{e}}\right\|_{\infty} \\
& \ell(x, u)=\max _{j \in \mathbb{N}_{[1, p]}}\left\{y_{j}-\left(y_{\mathrm{t}}\right)_{j}, 0\right\}+\gamma\left\|u-u_{\mathrm{e}}\right\|_{\infty} \\
& \ell(x, u)=\sum_{j=1}^{p} \max \left\{y_{j}-\left(y_{\mathrm{t}}\right)_{j}, 0\right\}+\gamma\left\|u-u_{\mathrm{e}}\right\|_{\infty},
\end{aligned}
$$


where $\gamma>0$ and $y=C \otimes x$. The stage cost (34) corresponds to $Y_{\mathrm{e}}=\left\{y_{\mathrm{e}}\right\}$, and (35) or (36) correspond to $Y_{\mathrm{e}}=\left\{y: y \leq y_{\mathrm{t}}\right\}$. In the stage cost 34 the first term penalizes the deviation of the output from the output equilibrium $y_{\mathrm{e}}$ while the second term penalizes the deviation of the input from the input equilibrium $u_{\mathrm{e}}$. The stage costs 35 and 36 have the following interpretation: the first term penalizes the tardiness with respect to the due dates while the second term penalizes the deviation of the input from the input equilibrium. From Proposition 4.1 it follows that the $\mathcal{K}$-function $\alpha$ of Property $\mathbf{P 2}$ ' is in these cases the identity function, i.e. $\alpha(x)=x$.

Using the same notations as in Section 4.1 we obtain the following corollary:

Corollary 4.4 Suppose there exists a vector $b \in \mathbb{R}^{p}$ such that $Y_{\mathrm{e}} \subseteq\left\{y \in \mathbb{R}^{p}: y \leq b\right\}$ and that Assumption A1 holds. Then, using in the optimal control problem 29 a stage cost that satisfies Properties P1'-P2' we obtain an MPC law $\kappa_{y}^{\mathrm{MPC}}$ for which the corresponding closed-loop buffer levels are bounded.

Proof Using the same arguments as in the proof of Theorem 4.2 it follows that

$$
\begin{aligned}
& \lim _{k \rightarrow \infty} \kappa_{y}^{\mathrm{MPC}}\left(\phi\left(k ; x, \kappa_{y}^{\mathrm{MPC}}\right)\right)=u_{\mathrm{e}} \\
& \lim _{k \rightarrow \infty} d_{\infty}\left(C \otimes \phi\left(k ; x, \kappa_{y}^{\mathrm{MPC}}\right), Y_{\mathrm{e}}\right)=0
\end{aligned}
$$

and that $\phi\left(k ; x, \kappa_{y}^{\mathrm{MPC}}\right)$ is bounded for all $k$ since the system is observable according to Assumption $\mathbf{A 1}$ and therefore the buffer levels remain bounded for any finite initial state $x \in X_{N}$.

Using similar arguments as in Remark 4.3 (i) we conclude that for the stage costs 35 or 36 the corresponding MPC optimization problem 29 can be recast as a linear program whenever Assumption $\mathbf{A 2}$ holds. For the stage cost (34) the optimization problem 29 can be recast as a mixed-integer linear program.

\section{Time-optimal controller for MPL systems}

Given a maximum horizon length $N_{\max }$ we now consider the problem of ensuring that the completion times after $N$ events, where $N=1,2, \cdots, N_{\max }$, are less than or equal to a specified target time T (i.e. $y(N) \leq \mathbf{T}$ ), using the "latest" controller that satisfies the input and state constraints 15 . Here "latest" means that the input times should be as large as possible (so in a manufacturing context we would feed the raw material as late as possible). Note that such a problem, but without considering constraints, was considered also in (Baccelli et al. 1992, Chapter 6) in terms of lattice theory. The time-optimal control problem in our setting is different from the classical one: we want to maximize $N$ instead of minimizing it; so in fact a better term would be "throughput-optimal" control.

The time-optimal control problem can be posed in terms of an optimization problem: given $x:=x(0)$, find

$$
N^{0}(x)=\max _{N \in \mathbb{N}_{[1, N \max ]}, \mathbf{u} \in \Pi_{N}^{\mathrm{T}}(x)} N
$$


where $\Pi_{N}^{\mathbf{T}}(x):=\left\{\mathbf{u}: H x_{i}+G u_{i} \leq h, \forall i \in \mathbb{N}_{[1, N]}, y_{N} \leq \mathbf{T}\right\}$ with $y_{N}=C \otimes x_{N}$. Moreover, since we aim for the latest input times, we want $u_{1}, \ldots, u_{N}$ to be as big as possible [see also (Baccelli et al. 1992, Chapter 6)]. We denote with $X_{N}^{\mathbf{T}}=\{x$ : $\left.\Pi_{N}^{\mathbf{T}}(x) \neq \varnothing\right\}$, i.e. the set of initial states such that after $N$ steps the trajectory is below the target time $\mathbf{T}$. It follows that

$$
N^{0}(x)=\max _{N}\left\{N \in \mathbb{N}_{\left[1, N_{\max }\right]}: x \in X_{N}^{\mathbf{T}}\right\} .
$$

Since we want the latest controller, a suitable choice of the stage cost is $\ell(x, u)=$ $-\sum_{j=1}^{m} u_{j}$.

The time-optimal controller is then implemented as follows:

(1) For each $N \in \mathbb{N}_{\left[1, N_{\max }\right]}$, solve the linear program

$$
\min _{\mathbf{u} \in \Pi_{N}^{\mathbf{T}}(x)}-\sum_{i=1}^{N} \sum_{j=1}^{m}\left(u_{i}\right)_{j} .
$$

(2) Determine $N^{0}(x)$ according to Eq. 37.

(3) Apply the control sequence $\mathbf{u}^{0}(x)$ corresponding to the prediction horizon $N^{0}(x)$.

The time-optimal control problem involves solving $N_{\max }$ linear programs in Step 1 above. The set $X_{N}$ has the following interpretation: the boundary of the polyhedron $X_{N}^{\mathbf{T}}$ represents the latest starting times such that after $N$ events the output is below the target time $\mathbf{T}$.

\section{Example}

Consider the manufacturing system of Fig. 1. It consists of three processing units. Raw material is fed to the first two units, processed and sent to the third unit where assembly takes place. Each unit can only start working on a new product if it has finished processing the previous product. We assume that each processing unit starts working as soon as all parts are available. We denote with $u_{\text {sys }}(k)$ the time at which a batch of raw material is fed to the system for the $k$ th cycle, $\left(x_{\mathrm{sys}}\right)_{i}(k)$ the time at which unit $i$ starts working for the $k$ th cycle, and $y_{\text {sys }}(k)$ the time at which the $k$ th

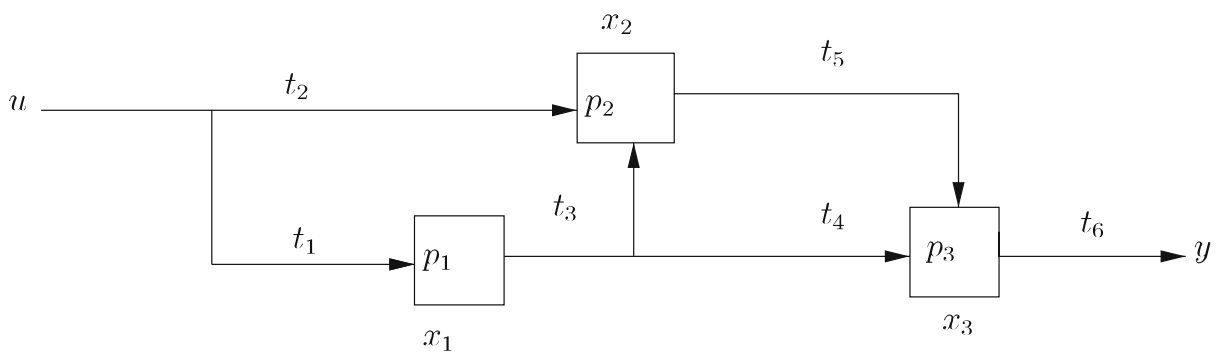

Fig. 1 A manufacturing system 
product leaves the system. We also denote with $p_{i}$ and $t_{j}$ the transportation times and processing times, respectively. We take the following value for these parameters: $t_{1}=1, t_{2}=1, t_{3}=0, t_{4}=3, t_{5}=0, t_{6}=0, p_{1}=1, p_{2}=2, p_{3}=2$.

Now we explain in more detail the dynamical equation that describes the evolution of the first processing unit: unit 1 will start with job $k$ when

- the previous job is finished, indicated by $\left(x_{\text {sys }}\right)_{1}(k-1)+p_{1}$ (i.e. the start of the previous job $(k-1)$ plus the production time $\left.p_{1}\right)$, and

- the raw material has arrived at the unit at time $u_{\text {sys }}(k)+t_{1}$ (i.e. the time the raw material is put into the system plus the transportation time $\left.t_{1}\right)$.

Since processing unit 1 starts working on as soon as the raw material is available and the current product has left the machine, this implies that we have $\left(x_{\text {sys }}\right)_{1}(k)=$ $\max \left\{\left(x_{\text {sys }}\right)_{1}(k-1)+1, u_{\text {sys }}(k)+1\right\}$. In max-plus algebra this expression can be written as $\left(x_{\text {sys }}\right)_{1}(k)=1 \otimes\left(x_{\text {sys }}\right)_{1}(k-1) \oplus 1 \otimes u_{\text {sys }}(k)$. The same reasoning applies to the second and third processing unit. Therefore, the MPA state space equations of the system, written in matrix form, are

$$
\begin{aligned}
& x_{\text {sys }}(k)=\left[\begin{array}{lll}
1 & \varepsilon & \varepsilon \\
2 & 2 & \varepsilon \\
5 & 4 & 2
\end{array}\right] \otimes x_{\mathrm{sys}}(k-1) \oplus\left[\begin{array}{l}
1 \\
2 \\
5
\end{array}\right] \otimes u_{\text {sys }}(k) \\
& y_{\mathrm{sys}}(k)=\left[\begin{array}{lll}
\varepsilon & \varepsilon & 2
\end{array}\right] \otimes x_{\mathrm{sys}}(k) .
\end{aligned}
$$

For this example the (maximal) MPA eigenvalue of the system matrix $A_{\text {sys }}$ is $\lambda_{\max }=2$. We consider the reference signal for the output $r_{\text {sys }}(k)=5+\rho k$ with $\rho=1.5 \lambda_{\max }=3$. We take the following constraints:

$$
\begin{gathered}
u_{\text {sys }}(k)-u_{\text {sys }}(k+1) \leq 0 \\
\left(x_{\text {sys }}\right)_{2}(k)-u_{\text {sys }}(k) \leq 2.5 .
\end{gathered}
$$

The initial conditions are $x_{\text {sys }}(0)=\left[\begin{array}{lll}9 & 13 & 14\end{array}\right]^{T}, u_{\text {sys }}(0)=6$.

We now apply MPC. We choose the prediction horizon $N=12$. We consider the stage cost 36 and we apply the MPC approach of Section 4.2. In this case the MPC optimization problem 29 can be recast as a linear program.

For the normalized system (obtained as in Section 2) the positively invariant set $\mathcal{O}_{\infty}$ is determined after 4 iterations: $\mathcal{O}_{\infty}=\mathcal{O}_{4}=\left\{x \in \mathbb{R}^{4}: I_{4} x \leq\left[\begin{array}{llll}0.5 & -0.5 & 0 & 0\end{array}\right]^{T}\right\}$.

By solving the linear program 29 in a receding horizon fashion we obtain for the original system the following MPC input sequence:

$$
\left\{\kappa_{y}^{\mathrm{MPC}}(x(k-1))+\rho k\right\}_{k=0}^{15}=6,12.5,14.5,16.5,18.5,20.5,22.5,24.5,
$$

$$
26.5,28.5,30.5,32.5,34.5,37,40,43 \text {. }
$$

The results of the closed-loop simulations are displayed in Fig. 2. We observe from the top plot that although we start later than the initial due date the closed-loop output is able to track the due date signal after a finite transient behavior, i.e. we have closed-loop stability. The middle plot displays the MPC input. We see that the 

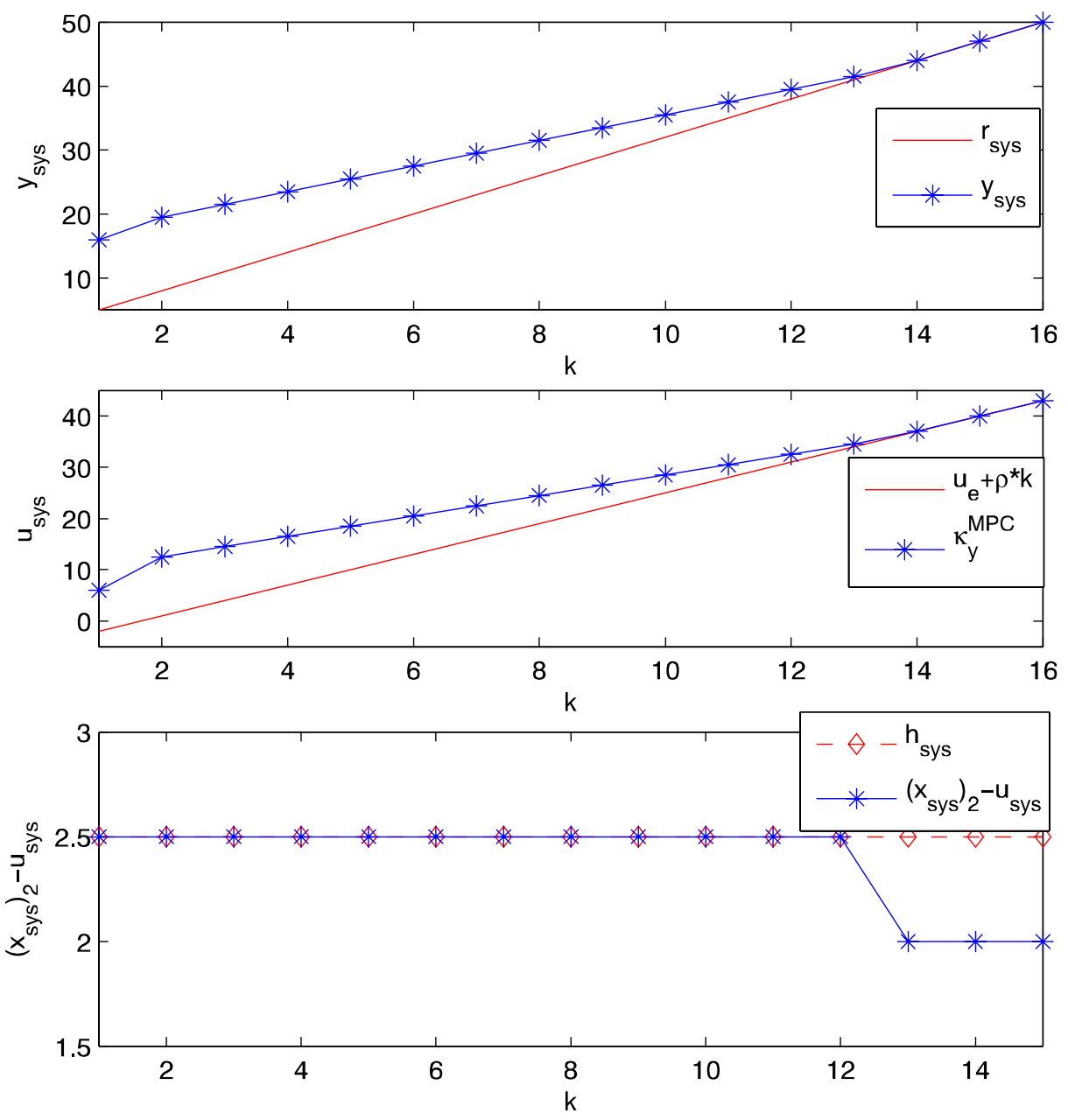

Fig. 2 The closed-loop MPC simulations

MPC input reaches the steady-state behavior in finite number of steps and that it is nondecreasing. The input-state constraints 39 are depicted in the bottom plot. Note that the MPC keeps the system behavior as close as possible to the constraints.

Let us now compare our MPC method with the other control design methods mentioned in Section 1. The max-plus control approaches proposed in (Baccelli et al. 1992; Cottenceau et al. 2001; Libeaut and Loiseau 1995; Menguy et al. 1997) typically involve an open-loop optimal control problem over a simulation horizon and for a given due date signal $r_{\text {sys }}$ such that the output of the system $y_{\text {sys }}$ should satisfy $y_{\text {sys }}(k) \leq r_{\text {sys }}(k)$ for all $k$. The solution of this optimal control problem is computed using residuation, resulting in a just-in-time control input. The main disadvantage of this approach is that it cannot cope with tracking problems where the outputs do not occur before the due dates, and that the resulting control input sequence is sometimes 
decreasing, i.e. the constraint 38 might be violated. For instance, if we apply the method of (Libeaut and Loiseau 1995) we get the following just-in-time control sequence $\left\{u_{\text {sys }}(k)\right\}_{k=0}^{15}=6,1,4,7,10,13,16,19,22,25,28,31,34,37,40,43$. This sequence is not feasible since we have $u_{\text {sys }}(1)=1<u_{\text {sys }}(0)=6$, i.e. the constraint 38 is violated. This infeasibility is caused by the fact that the optimal input aims to fulfill the constraint $y_{\text {sys }}(k) \leq r_{\text {sys }}(k)$ for all $k$, which cannot be met using a nondecreasing input sequence. So other residuation-based control design methods that also include this constraint such as (Baccelli et al. 1992; Cottenceau et al. 2001; Menguy et al. 1997) would also yield a control sequence that is not nondecreasing and thus infeasible.

These issues are overcome in (Maia et al. 2003; Menguy et al. 2000) by using a projection on the set of nondecreasing input sequences, or by considering a residuation-based adaptive control approach. The methods of (Maia et al. 2003; Menguy et al. 2000) result in nondecreasing input sequences and allow violations of the due dates. However, in contrast to the MPC approach presented in this paper the approaches of (Maia et al. 2003; Menguy et al. 2000) cannot cope with more complex state and input constraints, such as Eq. 39. For instance, using the adaptive control approach of (Menguy et al. 2000) we obtain the following optimal input sequence $\left\{u_{\text {sys }}(k)\right\}_{k=0}^{15}=6,6,6,7,10,13,16,19,22,25,28,31,34,37,40,43$. However, by applying this control the constraint 39 is violated (e.g. $\left.\left(x_{\text {sys }}\right)_{2}(1)-u_{\text {sys }}(1)=9 \not 2.5\right)$.

The MPC approach of De Schutter and van den Boom (2001) can cope with stateinput constraints. However, this approach cannot guarantee a priori stability of the closed-loop system. Note that stability is really an issue when designing controllers for MPL systems [see van den Boom et al. (2005) for an illustrative example where instability of the MPC-MPL closed-loop system occurs].

\section{Conclusions}

In this paper we have discussed the problem of stabilization of an MPL system subject to state-input constraints using MPC. We have derived an MPC strategy based on a terminal set-terminal cost approach that guarantees that the closed-loop input and state sequences satisfy a given set of linear inequality constraints. We have also shown that with this strategy asymptotic stability can be guaranteed a priori. For particular nonnegative piecewise affine stage costs we have shown that the MPLMPC optimization problem can be recast as a linear program for which efficient algorithms exist. Moreover, under some additional assumptions we have proved that two types of stability (asymptotic stability and boundedness) hold for the closed-loop MPC. For the time-optimal MPL control subject to linear constraints on the inputs and states we have also derived the solution, based on linear programming.

Acknowledgements Work supported by the STW projects "Model predictive control for hybrid systems" (DMR.5675) and "Multi-agent control of large-scale hybrid systems" (DWV.6188), and by the European projects HYCON "HYbrid CONtrol: Taming Heterogeneity and Complexity of Networked Embedded Systems" (FP6-IST-511368) and SICONOS "Modelling, Simulation and Control of Nonsmooth Dynamical Systems" (IST-2001-37172).

The authors also want to thank the anonymous reviewers of this paper for their useful suggestions and comments, and for pointing out an incompleteness in the original proof of Proposition 4.1. 


\section{References}

Baccelli F, Cohen G, Olsder GJ, Quadrat JP (1992) Synchronization and linearity. Wiley, New York

Cofer DD, Garg VK (1996) Supervisory control of real-time discrete-event systems using lattice theory. IEEE Trans Automat Contr 41(2):199-209, February

Cottenceau B, Hardouin L, Boimond J, Ferrier J (2001) Model reference control for timed event graphs in dioid. Automatica 37(8):1451-1458

Cuninghame-Green RA (1979) Minimax algebra, Lecture notes in economics and mathematical systems, vol 16. Springer, Berlin, Germany

De Schutter B (1996) Max-algebraic system theory for discrete event systems. PhD thesis, Faculty of Applied Sciences, K.U.Leuven, Leuven, Belgium

De Schutter B, van den Boom T (2001) Model predictive control for max-plus-linear discrete event systems. Automatica 37(7):1049-1056, July

Elsner L, Johnson CR, Dias da Silva JA (1988) The Perron root of a weighted geometric mean of nonnegative matrices. Linear Multilinear Algebra 24(1):1-13

Gaubert S (1996) On the Burnside problem for semigroups of matrices in the $(\max ,+)$ algebra. Semigroup Forum 52:271-292

Gazarik MJ, Kamen BEW (1999) Reachability and observability of linear systems over max-plus. Kybernetika 35(1):2-12, January

Gilbert EG, Tan KT (1991) Linear systems with state and control constraints: the theory and applications of maximal output admissible sets. IEEE Trans Automat Contr 36(9):1008-1020, September

Heemels WPMH, De Schutter B, Bemporad A (2001) Equivalence of hybrid dynamical models. Automatica 37(7):1085-1091, July

Heidergott B, Olsder GJ, Woude J (2005) Max plus at work. Princeton University Press, Princeton

Kumar R, Garg VK (1994) Extremal solutions of inequations over lattices with applications to supervisory control. In: Proceedings of the 33rd IEEE conference on decision and control. Orlando, Florida, pp 3636-3641, December

Libeaut L, Loiseau JJ (1995) Admissible initial conditions and control of timed event graphs. In: Proceedings of the 34th IEEE conference on decision and control. New Orleans, Louisiana, pp 2011-2016, December

Maciejowski JM (2002) Predictive control with constraints. Prentice Hall, Harlow, England

Maia CA, Hardouin L, Santos-Mendes R, Cottenceau B (2003) Optimal closed-loop control of timed event graphs in dioids. IEEE Trans Automat Contr 48(12):2284-2287, December

Mairesse J (1995) A graphical approach to the spectral theory in the (max,+) algebra. IEEE Trans Automat Contr 40(10):1783-1789, October

Mayne DQ, Rawlings JB, Rao CV, Scokaert POM (2000) Constrained model predictive control: stability and optimality. Automatica 36(7):789-814, June

Menguy E, Boimond JL, Hardouin L (1997) A feedback control in max-algebra. In: Proceedings of the european control conference (ECC'97), Brussels, Belgium, paper 487, July

Menguy E, Boimond JL, Hardouin L, Ferrier JL (2000) A first step towards adaptive control for linear systems in max algebra. Discret Event Dyn Syst Theor Appl 10(4):347-367

Necoara I (2006) Model predictive control for max-plus-linear and piecewise affine systems. PhD thesis, Delft Center for Systems and Control, Delft University of Technology, The Netherlands, October

Necoara I, van den Boom TJJ, De Schutter B, Hellendoorn J (2006) Stabilization of MPL systems using model predictive control: the unconstrained case. Technical Report 06-006, Delft Center for Systems and Control, Delft University of Technology, Delft, The Netherlands, Revised version. Provisionally accepted for Automatica, September

Passino KM, Burgess KL (1998) Stability analysis of discrete event systems. Wiley, New York

La Salle JP (1976) The stability of dynamical systems. Society for Industrial and Applied Mathematics, Philadelphia, PA

van den Boom TJJ, De Schutter B, Necoara I (2005) On MPC for max-plus-linear systems: analytic solution and stability. In: Proceedings of the 44th IEEE conference on decision and control, and the european control conference, (CDC-ECC'05). Seville, Spain, pp 7816-7821, December 


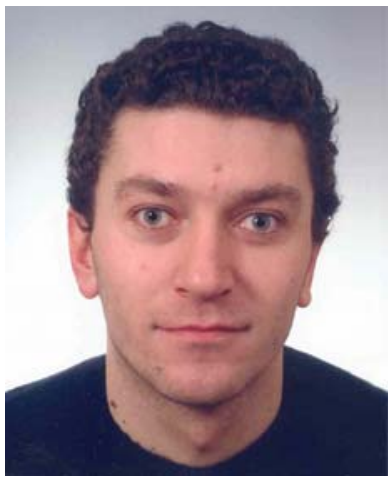

Ion Necoara received the MSc degree in optimization and statistics from University of Bucharest, Romania in 2002 and the PhD degree in Applied Sciences (summa cum laude) from Delft University of Technology, The Netherlands in 2006. Currently, he is a postdoctoral research associate at the Electrical Engineering Department (ESAT-SCD), K.U. Leuven, Belgium. His current research interests include distributed and cooperative optimization for large scale problems, hybrid systems control, distributed receding horizon control.

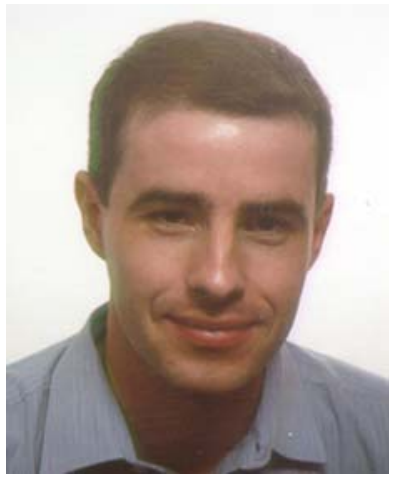

Bart De Schutter received the MSc degree in Electrotechnical-Mechanical Engineering in 1991 and the PhD degree in Applied Sciences (summa cum laude with congratulations of the examination jury) in 1996, both at K. U. Leuven, Belgium. Currently, he is a full professor at the Delft Center for Systems and Control and at the Marine \& Transport Technology department of Delft University of Technology in Delft, The Netherlands. Bart De Schutter was awarded the 1998 SIAM Richard C. DiPrima Prize and the 1999 K.U. Leuven Robert Stock Prize for his PhD thesis. His current research interests include hybrid systems control, discrete-event systems, multi-agent systems, control of intelligent transportation systems, and optimization. 


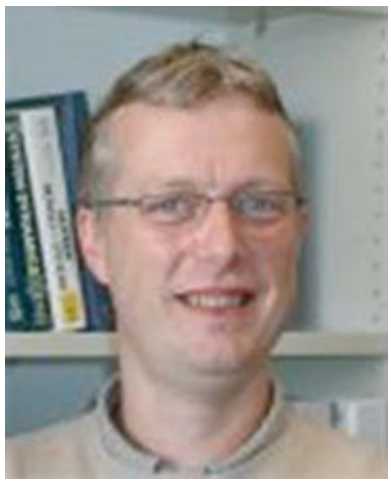

Ton J. J. van den Boom was born in Bergen OP Zoom (The Netherlands). He received his MSc and $\mathrm{PhD}$ degrees in Electrical Engineering from the Eindhoven University of Technology, The Netherlands, in 1988 and 1993, respectively. Currently he is an Associate Professor at the Delft University of Technology, The Netherlands. His research interests are in the areas of linear and nonlinear model predictive control, and control of discrete event systems and hybrid systems.

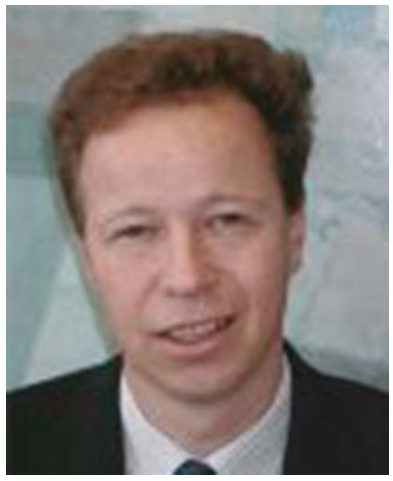

Hans Hellendoorn was born in Rotterdam, The Netherlands, in 1964. He studied Computer Science at Delft University of Technology in Delft, The Netherlands. Next, he did research in fuzzy logic in Delft and at the National Aerospace Laboratory in Amsterdam resulting, in 1990, in a PhD-thesis with the title "Reasoning with Fuzzy Logic."

He started 1991 at the Siemens Research Laboratory in Munich in the fuzzy control group that he leaded until 1997. From 1997 to 2000 he was department head for training simulation at Siemens in the Netherlands, since 2001 he is responsible for innovation management. Since 1998, he is part-time professor for control theory at the Delft University of Technology.

Hans Hellendoorn has written several books and a large number of publications on computational intelligence and control. He currently works on traffic control and hybrid and multi-agent systems. 